\title{
RAUL OSSADA
}

Modelagem da dinâmica de doenças infecciosas em redes de movimentação de animais

São Paulo 



\section{RAUL OSSADA}

Modelagem da dinâmica de doenças infecciosas em redes de movimentação de animais

Dissertação apresentada ao Programa de Pós-Graduação em Epidemiologia Experimental Aplicada às Zoonoses da Faculdade de Medicina Veterinária e Zootecnia da Universidade de São Paulo, para obtenção do título de Mestre em Ciências

\section{Departamento:}

Medicina Veterinária Preventiva e Saúde Animal

\section{Área de Concentração:}

Epidemiologia Experimental Aplicada às Zoonoses

\section{Orientador:}

Prof. Dr. Marcos Amaku

São Paulo 
Autorizo a reprodução parcial ou total desta obra, para fins acadêmicos, desde que citada a fonte.

DADOS INTERNACIONAIS DE CATALOGAÇÃO-NA-PUBLICAÇÃO

(Biblioteca Virginie Buff D’Ápice da Faculdade de Medicina Veterinária e Zootecnia da Universidade de São Paulo)

Ossada, Raul

Modelagem da dinâmica de doenças infecciosas em redes de movimentação de

FMVZ animais / Raul Ossada. -- 2011

$43 \mathrm{f}$

Dissertação (Mestrado) - Universidade de São Paulo. Faculdade de Medicina Veterinária e Zootecnia. Departamento de Medicina Veterinária Preventiva e Saúde Animal, São Paulo, 2011

Zoonoses.

Programa de Pós-Graduação: Epidemiologia Experimental Aplicada às

Área de concentração: Epidemiologia Experimental Aplicada às Zoonoses.

Orientador: Prof. Dr. Marcos Amaku.

1. Redes complexas. 2. Movimentação de animais. 3. Bovinos. 4. Modelagem epidemiológica. 5. Espalhamento de doenças. I. Título. 
UNIVERSIDADE DE SÃO PAULO

\section{Comissão de Ética para uso de animais}

\section{CERTIFICADO}

Certificamos que o Projeto intitulado "Modelagem da dinâmica de doenças infecciosas em redes de movimentação de animais", protocolado sob o n'1819/2009, não utilizando animais, sob a responsabilidade do Prof. Dr. Marcos Amaku, está de acordo com os princípios éticos de experimentação animal da "Comissão de Ética para uso de animais" da Faculdade de Medicina Veterinária e Zootecnia da Universidade de São Paulo e foi aprovado em reunião de 25/11/2009.

We certify that the Research "Modeling the dynamics of infectious diseases in networks of animal movements", protocol number 1819/2009, will not be utilized animals, under the responsibility Prof. Dr. Marcos Amaku, agree with Ethical Principles in Animal Research adopted by "Ethics Committee for animal use" of the School of Veterinary Medicine and Animal Science of University of São Paulo. was approved in the meeting of day 11/25/2009.

São Paulo, 30 de novembro de 2009

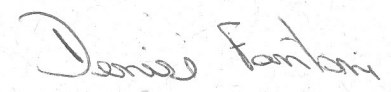

Profa Dra Denise Tabacchi Fantoni Presidente 


\section{FOLHA DE AVALIAÇÃO}

Nome: OSSADA, Raul

Título: Modelagem da dinâmica de doenças infecciosas em redes de movimentação de animais

Dissertação apresentada ao Programa de PósGraduação em Epidemiologia Experimental Aplicada às Zoonoses da Faculdade de Medicina Veterinária e Zootecnia da Universidade de São Paulo, para obtenção do título de Mestre em Ciências

Data:

\section{Banca Examinadora}

Prof. Dr.

Instituição:

Assinatura:

Julgamento:

Prof. Dr.

Instituição:

Assinatura:

Julgamento:

Prof. Dr.

Instituição:

Assinatura:

Julgamento: 


\section{DEDICATÓRIA}

Dedico este trabalho à minha mãe Yoko Saito Ossada, ao meu pai Oswaldo Ossada e ao meu irmão Helder Ossada por sempre estarem ao meu lado nos momentos de maior dificuldade, mas principalmente por estarem presentes nos momentos de maior alegria. 


\section{AGRADECIMENTOS}

Agradeço ao Professor Doutor Marcos Amaku, por ter me orientado durante este trabalho, me dando o tempo necessário para me acostumar com a forma de trabalho de um pesquisador, permitindo que eu adquirisse experiência tanto dos meus erros quanto dos meus acertos.

Agradeço aos membros da banca, Professor Doutor Fernando Ferreira e Professora Doutora Clarice Gameiro da Fonseca Pachi por aceitarem o convite de participarem da banca e pela contribuição fornecida para o enriquecimento do trabalho, permitindo que eu enxergasse coisas que não me eram claro antes.

Agradeço aos amigos José Henrique de Hildebrand e Grisi Filho e a Doutora Rísia Lopes Negreiros pela ajuda direta neste trabalho, sempre dispostos a me ajudarem em todos os momentos.

Agradeço a todos do Laboratório de Epidemiologia e Bioestatística (LEB) pelo companherismo, carinho e amizade durante os momentos mais críticos do trabalho.

Agradeço aqueles que me ajudaram direta ou indiretamente na conclusão deste trabalho, pois foi com a colaboração de vocês que este trabalho chegou ao seu fim.

Agradeço, enfim, a você que, por qualquer que seja o motivo, está lendo este trabalho. 


\section{RESUMO}

OSSADA R. Modelagem da dinâmica de doenças infecciosas em redes de movimentação de animais. [Modeling the dynamics of infectious diseases in networks of animal movements]. 2011. 43 p. Dissertação (Mestrado em Ciências) - Faculdade de Medicina Veterinária e Zootecnia, Universidade de São Paulo, São Paulo, 2011.

A dinâmica de movimentação de animais em uma rede de propriedades rurais e o espalhamento de algumas doenças animais estão intrinsecamente relacionados. Assim, compreender a dinâmica do espalhamento de doenças infecciosas nestas redes é um instrumento importante no controle destas. Neste projeto, foram implementados algoritmos para gerar redes de movimentação de animais hipotéticas e reconstruiu-se a rede de movimentações de bovinos do Estado do Mato Grosso, 2007, Brasil. Foram feitas diversas simulações a fim de verificar o espalhamento de doenças agudas e crônicas nessas redes. Diferentes dinâmicas de espalhamento de doenças infecciosas foram observadas em redes com a mesma distribuição de graus e diferentes estruturas topológicas. Espera-se que os resultados das simulações matemáticas possam auxiliar nas atividades dos órgãos responsáveis pela vigilância epidemiológica e incentivar outros Estados a seguirem o exemplo do Estado do Mato Grosso, a construírem bancos de dados que possam ser analisados utilizando a metodologia de redes.

Palavras-chave: Redes complexas. Movimentação de animais. Bovinos. Modelagem epidemiológica. Espalhamento de doenças. 


\begin{abstract}
OSSADA R. Modeling the dynamics of infectious diseases in networks of animal movements. [Modelagem da dinâmica de doenças infecciosas em redes de movimentação de animais]. 2011. 43 p. Dissertação (Mestrado em Ciências) - Faculdade de Medicina Veterinária e Zootecnia, Universidade de São Paulo, São Paulo, 2011.

The animals' movements in a farms network and the spread of some animal diseases are intrinsically related. Therefore, comprehending the dynamics of the spreading of infectious diseases in these networks is an important tool in controlling these diseases. In this project, we have implemented algorithms to generate hypothetical networks of animals' movements and rebuilt the network of bovine movements from the State of Mato Grosso, 2007, Brazil. We made several simulations in order to check the spreading of acute and chronic disease in these networks. Different dynamics of infectious disease spreading were observed in networks with the same degree distribution and different topological structure. We hope that the results of the mathematical simulations may assist in the activities of agencies responsible for disease surveillance and encourage other States to follow the example of the State of Mato Grosso, to build databases that can be analyzed using the methodology of networks.
\end{abstract}

Keywords: Complex networks. Animal movements. Cattle. Epidemic modeling. Disease spreading. 


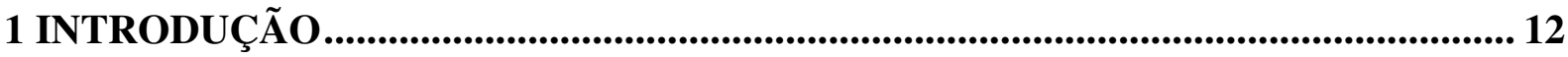

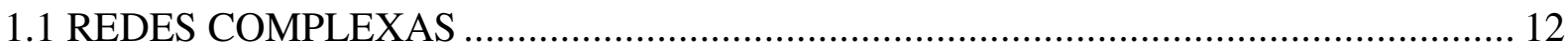

1.2 MODELAGEM DO ESPALHAMENTO DE DOENÇAS EM REDES ......................... 13

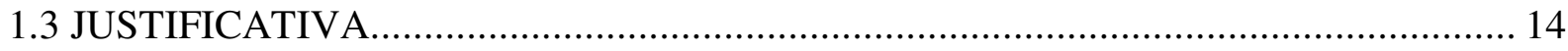

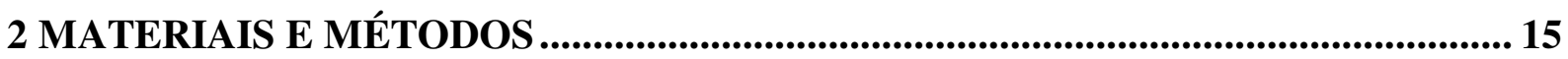

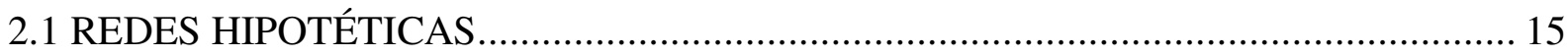

2.1.1 Modelo Barabási-Albert................................................................................... 16

2.1.2 Modelo Método A ............................................................................................ 17

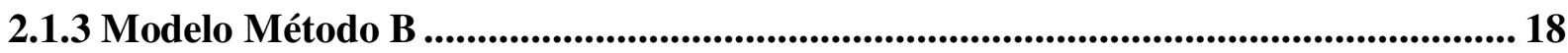

2.1.4 Modelo Kalisky ............................................................................................................. 19

2.1.5 Modelo Molloy-Reed .................................................................................................................. 20

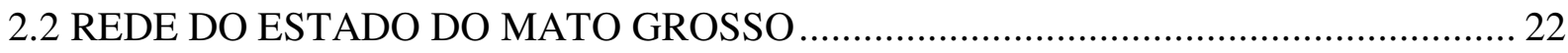

2.3 MODELO DAS SIMULAÇÕES DA DINÂMICA DE ESPALHAMENTO.................. 23

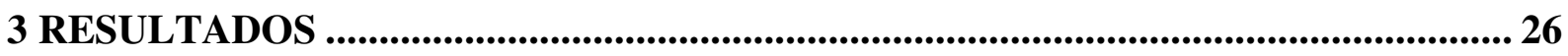

3.1 SIMULAÇÕES NAS REDES NÃO DIRECIONADAS ............................................. 26

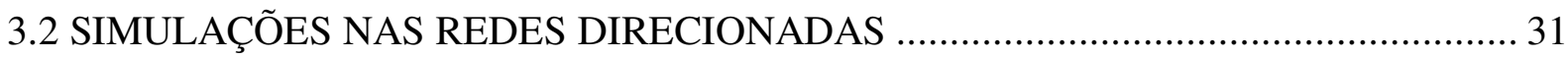

3.3 SIMULAÇÕES NA REDE DO MATO GROSSO................................................. 36

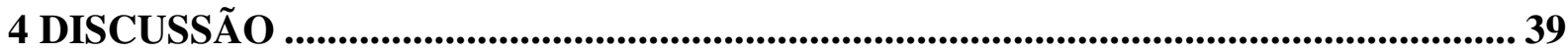

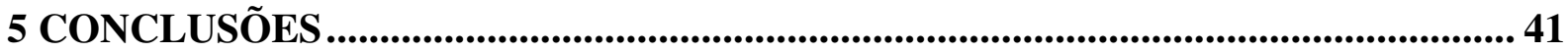

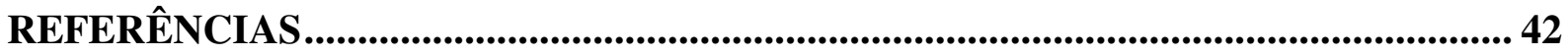




\section{INTRODUÇÃO}

Nesta seção, apresentamos uma breve descrição dos temas abordados neste projeto, além de uma justificativa para o mesmo.

\subsection{REDES COMPLEXAS}

Diversos sistemas biológicos, sociais e de comunicação podem ser descritos por redes complexas (PASTOR-SATORRAS; VESPIGNANI, 2001), cujos vértices representam indivíduos ou organizações, e as arestas representam as conexões ou as interações entre eles. Tais arestas podem ser: não direcionadas, representando, por exemplo, relações sexuais entre portadores do vírus HIV (caracterizando uma rede não direcionada); ou direcionadas, representando, por exemplo, a venda de um lote de bovinos de uma fazenda A para outra fazenda B (caracterizando assim uma rede direcionada). Temos também, em algumas redes, a presença de arestas múltiplas, ou seja, quando existe mais de uma aresta entre o mesmo par de vértices, e laços, ou seja, quando uma aresta conecta um vértice a si mesmo (Figura 1).

Define-se também, que o grau $(k)$ de um vértice é o número de arestas que este possui. Sendo que, no caso de uma rede direcionada, define-se também o grau de entrada $\left(k_{i n}\right)$ de um vértice, como o número de arestas que apontam para este vértice; e o grau de saída $\left(k_{\text {out }}\right)$ de um vértice, como o número de arestas que partem deste vértice (WASSERMAN; FAUST, 1994; NEWMAN, 2010).
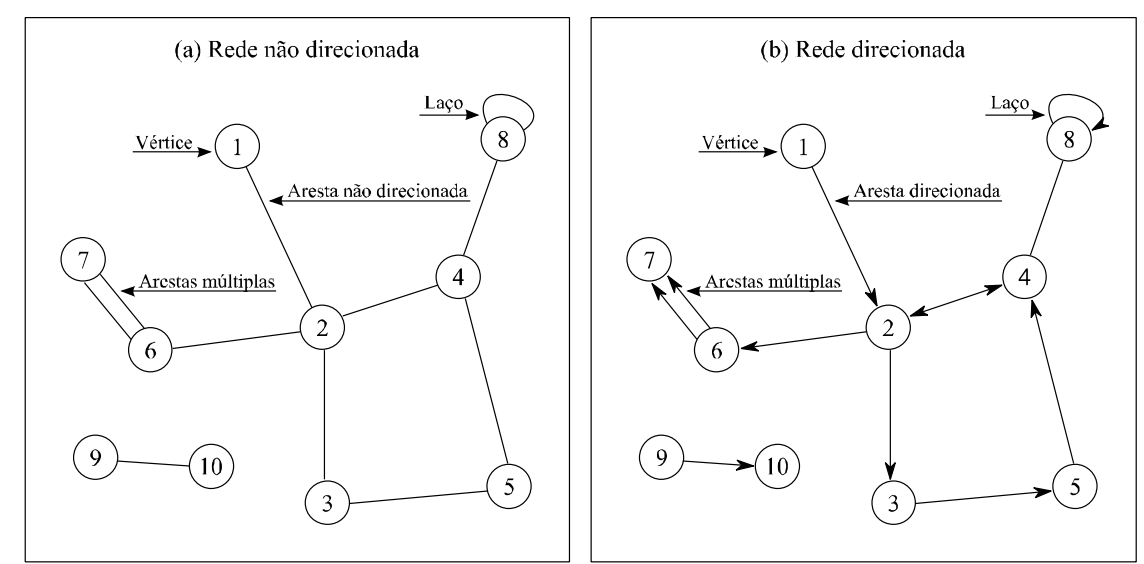

Figura 1 - (a) Rede cujas arestas são não direcionadas. (b) Mesma rede, mas cujas arestas são direcionadas. 
Sabe-se também que a probabilidade de um vértice destas redes ter $\mathrm{k}$ arestas pode seguir uma distribuição de graus lei de potência: $P(k) \sim k^{-\alpha}$, onde $\alpha$ é o expoente desta distribuição, cujo valor para tais redes geralmente varia de 2 até 3 (BARABÁSI; ALBERT, 1999; PASTOR-SATORRAS; VESPIGNANI, 2001; JEONG, 2003); caracterizando assim uma rede complexa livre de escala, ou simplesmente, rede complexa. Assim, a análise do $P(k)$ em função de $k$, pode indicar se uma rede pertence a uma categoria de redes complexas com características conhecidas ou não (Figura 2).

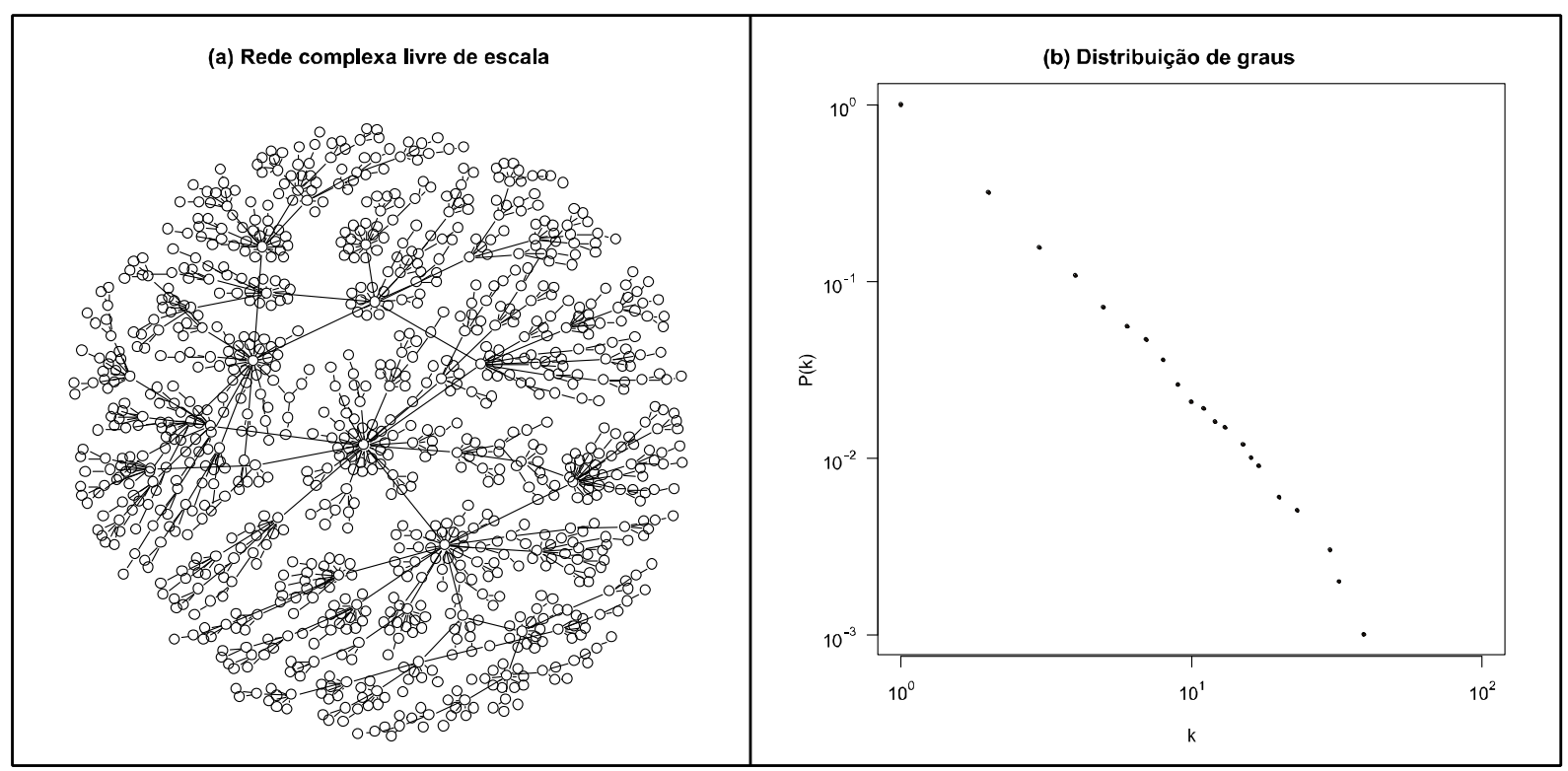

Figura 2 - (a) Ilustração de uma rede complexa livre de escala. (b) Distribuição de graus da rede apresentada.

Além do que já foi definido, quando se estuda redes complexas considerando de um período de tempo, podemos considerar a rede como sendo: estática, ou seja, que as arestas entre os vértices são permanentes ao longo do período estudado; ou dinâmica, ou seja, as arestas entre os vértices podem se alterar ao longo do período de estudo.

\subsection{MODELAGEM DO ESPALHAMENTO DE DOENÇAS EM REDES}

Modelos epidemiológicos tradicionais, geralmente, assumem que os indivíduos distribuem-se homogeneamente na população, isto é, cada indivíduo tem a mesma chance de encontrar-se com qualquer outro indivíduo. Todavia, tal suposição nem sempre é realista, pois sabe-se que há um alto grau de heterogeneidade na relação entre os indivíduos da população, 
ou seja, um indivíduo tem mais chances de se encontrar com outros que participem do mesmo grupo social que o seu. Assim, o uso das redes de contato acaba sendo um modelo mais realístico das interações entre os indivíduos na população (LINDQUIST et al., 2011).

Em virtude disto, vários estudos têm sido desenvolvidos a respeito do espalhamento de doenças usando a metodologia de redes (LLOYD; MAY, 2001; MAY; LLOYD, 2001; PASTOR-SATORRAS; VESPIGNANI, 2001; WOOLHOUSE et al., 2005; OSSADA et al., 2010). No caso de uma rede de movimentação de animais, os vértices representam as fazendas e as arestas representam a movimentação dos animais entre elas.

Como estas redes de movimentação animal apresentam um alto grau de heterogeneidade (BIGRAS-POULIN et al., 2006; BIGRAS-POULIN et al., 2007; NATALE et al., 2009), isto pode influenciar o potencial risco de transmissão de uma doença, como por exemplo no espalhamento da febre aftosa e da brucelose bovina. Assim, simular o espalhamento de doenças nestas redes pode indicar caminhos para a prevenção e o controle de doenças nos animais.

\subsection{JUSTIFICATIVA}

A modelagem do modo de transmissão é crucial, uma vez que os resultados de estratégias de controle podem variar muito, dependendo do modo como a infecção é transmitida na população. Além disso, ao se utilizar modelos matemáticos que procuram prever o padrão do espalhamento de uma doença na população, é possível simular uma série de cenários que na prática seriam inviáveis ou antiéticos, já que não é possível introduzir intencionalmente doenças nas populações ou negar uma intervenção que potencialmente salvaria uma vida em prol de um estudo científico (MEYERS, 2007).

Neste projeto, foram implementadas rotinas computacionais para a geração de redes complexas teóricas, que representariam redes de movimentação de animais entre propriedades, e rotinas para simular o espalhamento de doenças infecciosas hipotéticas com características agudas e crônicas. Além disso, foram usados os dados referentes à movimentação de bovinos no Estado do Mato Grosso, Brasil, no ano de 2007 (MATO GROSSO, 2008; NEGREIROS, 2010). 


\section{MATERIAIS E MÉTODOS}

Nesta seção, descrevemos como foram geradas as redes de movimentação de animais hipotéticas e a rede real do Estado do Mato Grosso. Também descrevemos o modelo usado nas simulações de espalhamento de doenças infecciosas.

\subsection{REDES HIPOTÉTICAS}

A seguir, apresentamos uma breve descrição dos algoritmos usados para a geração das redes complexas livre de escala hipotéticas, geradas com o intuito de testar as simulações epidemiológicas em redes com características conhecidas e verificar se alguma delas reproduziria os resultados das simulações epidemiológicas na rede do Estado do Mato Grosso. Estes modelos foram implementados utilizando-se o programa R (R DEVELOPMENT CORE TEAM, 2010) e o pacote igraph (CSARDI; NEPUSZ, 2006), desconsiderando-se neles arestas múltiplas (agrupando-os numa única aresta) e laços (descartando-os). 


\subsubsection{Modelo Barabási-Albert}

O algoritmo deste modelo é descrito, em mais detalhes, em (BARABÁSI; ALBERT, 1999), sendo representada na figura 3 uma rede que segue este modelo:

1. Inicia-se com um pequeno número $\left(m_{0}\right)$ de vértices desconectados.

2. A cada passo de tempo, acrescenta-se um novo vértice com $m\left(\leq m_{0}\right)$ arestas que conectam o novo vértice a $m$ diferente vértices que já existem na rede.

3. Para se escolher os vértices ao qual o novo vértice irá se conectar, assumimos que a probabilidade $p$ de que o novo vértice se conectará ao vértice $i$ depende do grau $k_{i}$, do vértice $i$, tal que: $p\left(k_{i}\right)=\frac{k_{i}}{\sum_{i} k_{i}}$

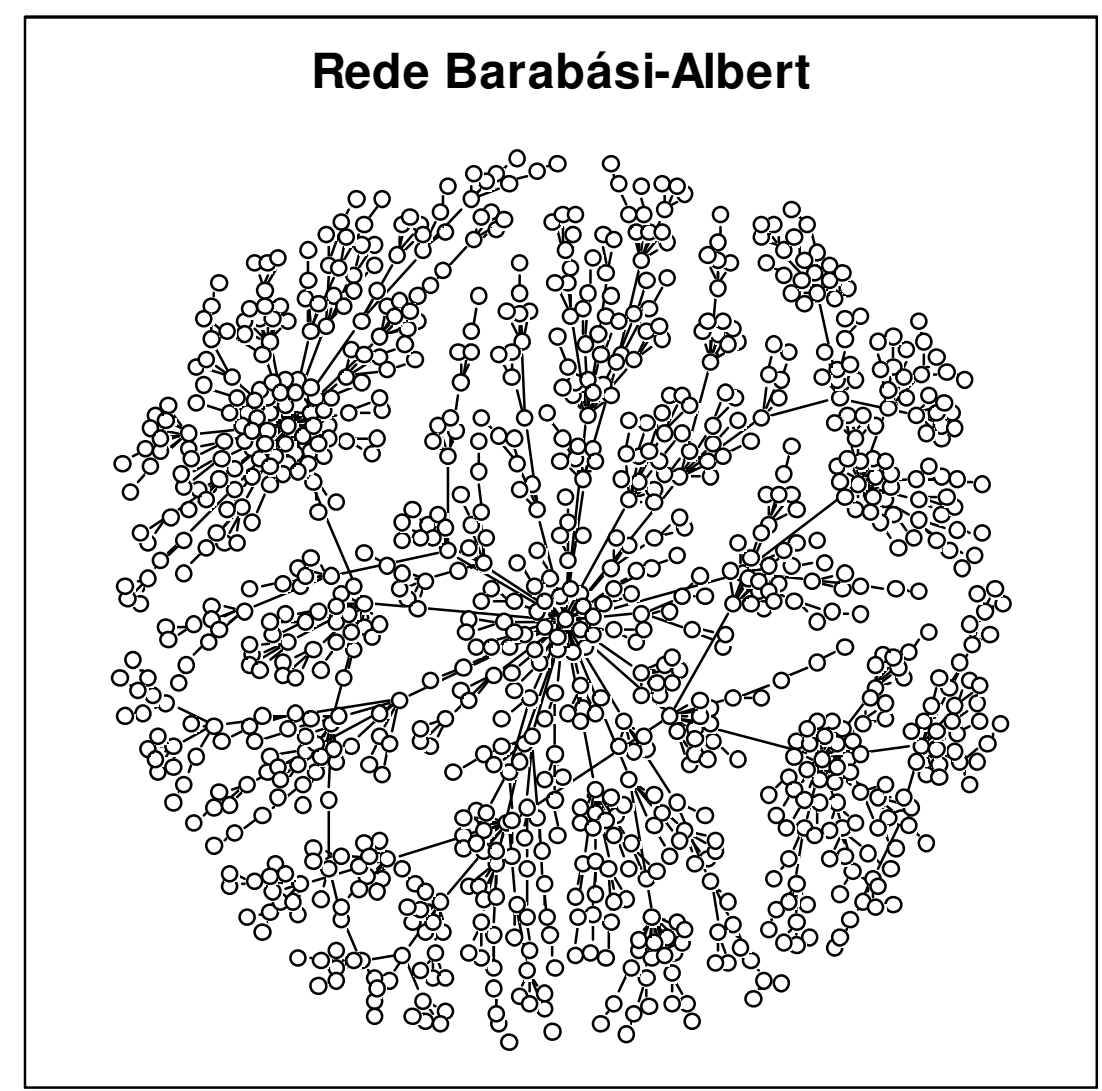

Figura 3 - Ilustração de uma rede que segue o modelo Barabási-Albert, com 1000 vértices e $m=1$ 


\subsubsection{Modelo Método A}

Este modelo é melhor detalhado em (GRISI-FILHO et al., 2010; GRISI-FILHO et al., em fase de elaboração ${ }^{1}$ ), sendo representada na figura 4 uma rede que segue este modelo:

1. Cada vértice recebe um grau da distribuição.

2. Escolhe-se o vértice com o maior grau $(K)$, conectando-o aleatoriamente à $K$ vértices diferentes.

3. Repete-se este processo até que não existam mais vértices com conexões a serem realizadas.

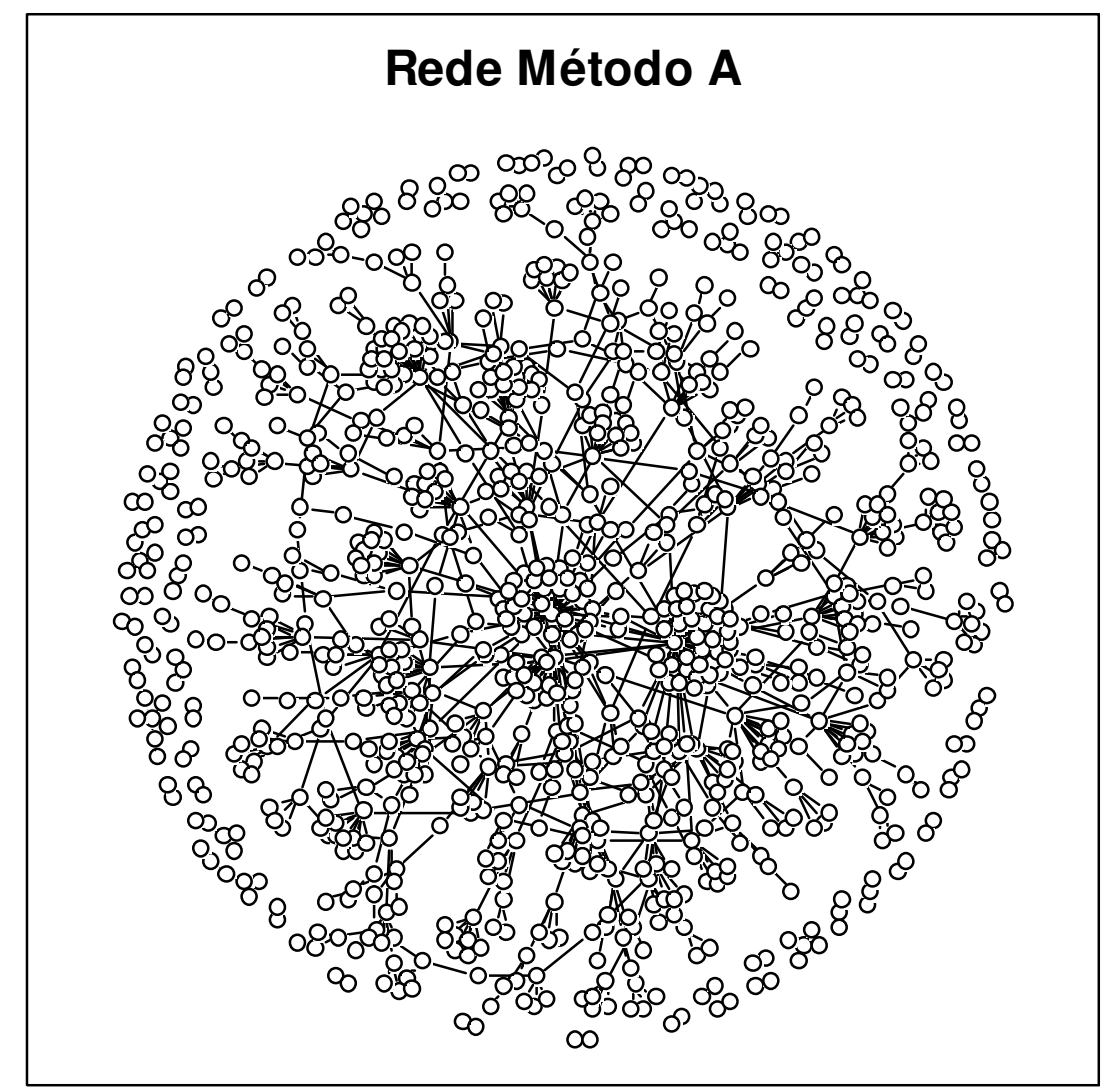

Figura 4 - Ilustração de uma rede que segue o modelo Método A, com $P(k)$ gerado a partir de uma rede Barabási-Albert com: vértices $=1000$ e $m=1$

\footnotetext{
${ }^{1}$ GRISI-FILHO, J. H. H.; OSSADA, R.; FERREIRA, F.; AMAKU, A. Different structural properties in scale-free networks with the same degree distribution.
} 


\subsubsection{Modelo Método B}

Este modelo é melhor descrito em (GRISI-FILHO et al., 2010; GRISI-FILHO et al., em fase de elaboração ${ }^{1}$ ), sendo representada na figura 5 uma rede que segue este modelo:

1. Cada vértice recebe um grau da distribuição.

2. Escolhe-se o vértice com o maior grau $(K)$, conectando-o aos primeiros $K$ vértices com conexões a serem realizadas.

3. Repete-se este processo até que não existam mais vértices com conexões a serem realizadas.

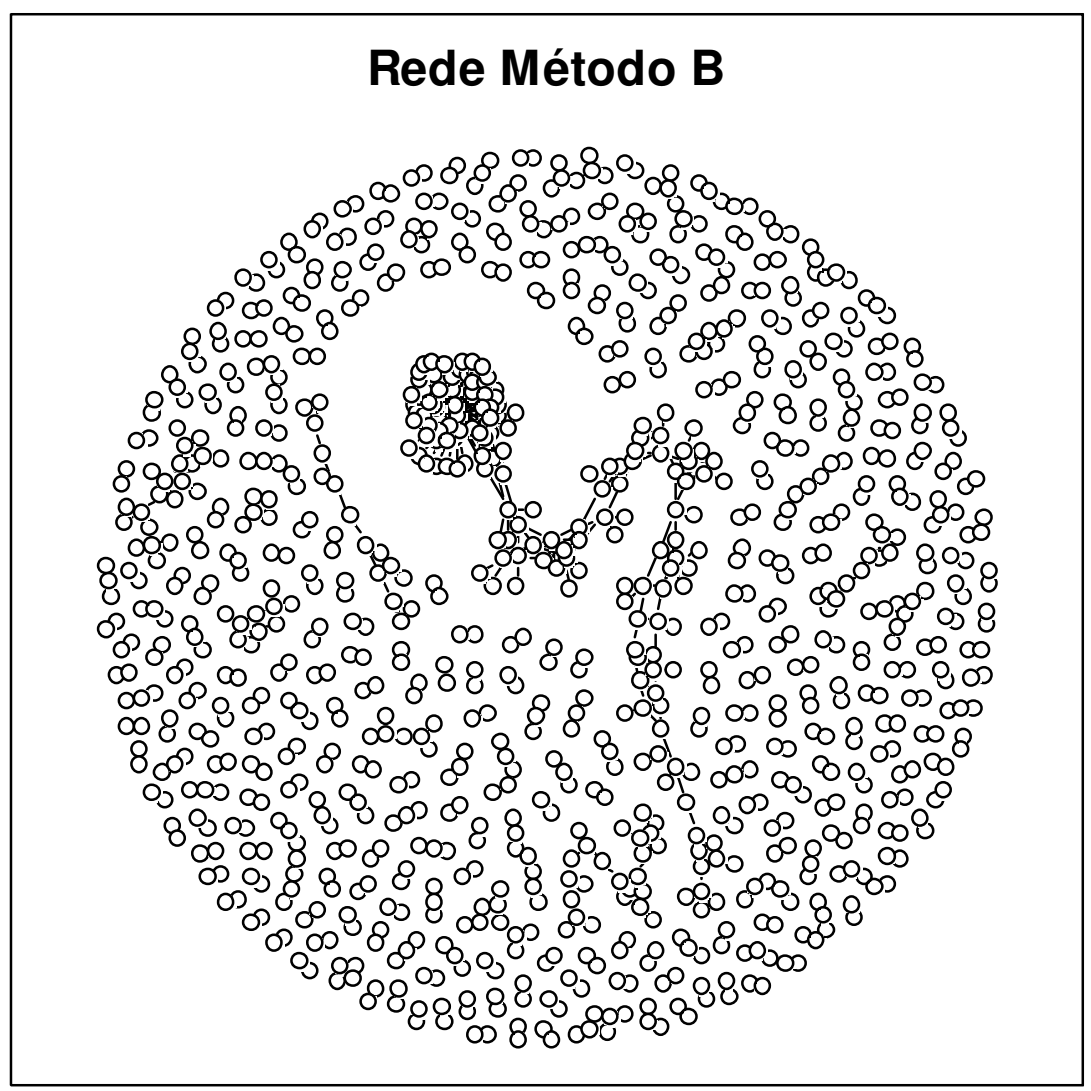

Figura 5 - Ilustração de uma rede que segue o modelo Método B, com $P(k)$ gerado a partir de uma rede Barabási-Albert com: vértices $=1000$ e $m=1$ 


\subsubsection{Modelo Kalisky}

Uma descrição mais detalhada deste algoritmo pode ser obtida em (KALISKY; COHEN; HAVLIN, 2004), sendo representada na figura 6 uma rede que segue este modelo:

1. Cada vértice recebe um grau da distribuição.

2. Escolhe-se o vértice com maior grau $(K)$, conectando-o aleatoriamente à $K$ vértices diferentes. O conjunto formado por este vértice e seus vizinhos é a $1^{\mathrm{a}}$ camada.

3. Forma-se a $2^{\mathrm{a}}$ camada conectando aleatoriamente todas as arestas dos vértices da $1^{\mathrm{a}}$ camada com vértices que ainda possuem conexões a serem realizadas.

4. Este processo é repetido até que não existam mais conexões a serem realizadas.

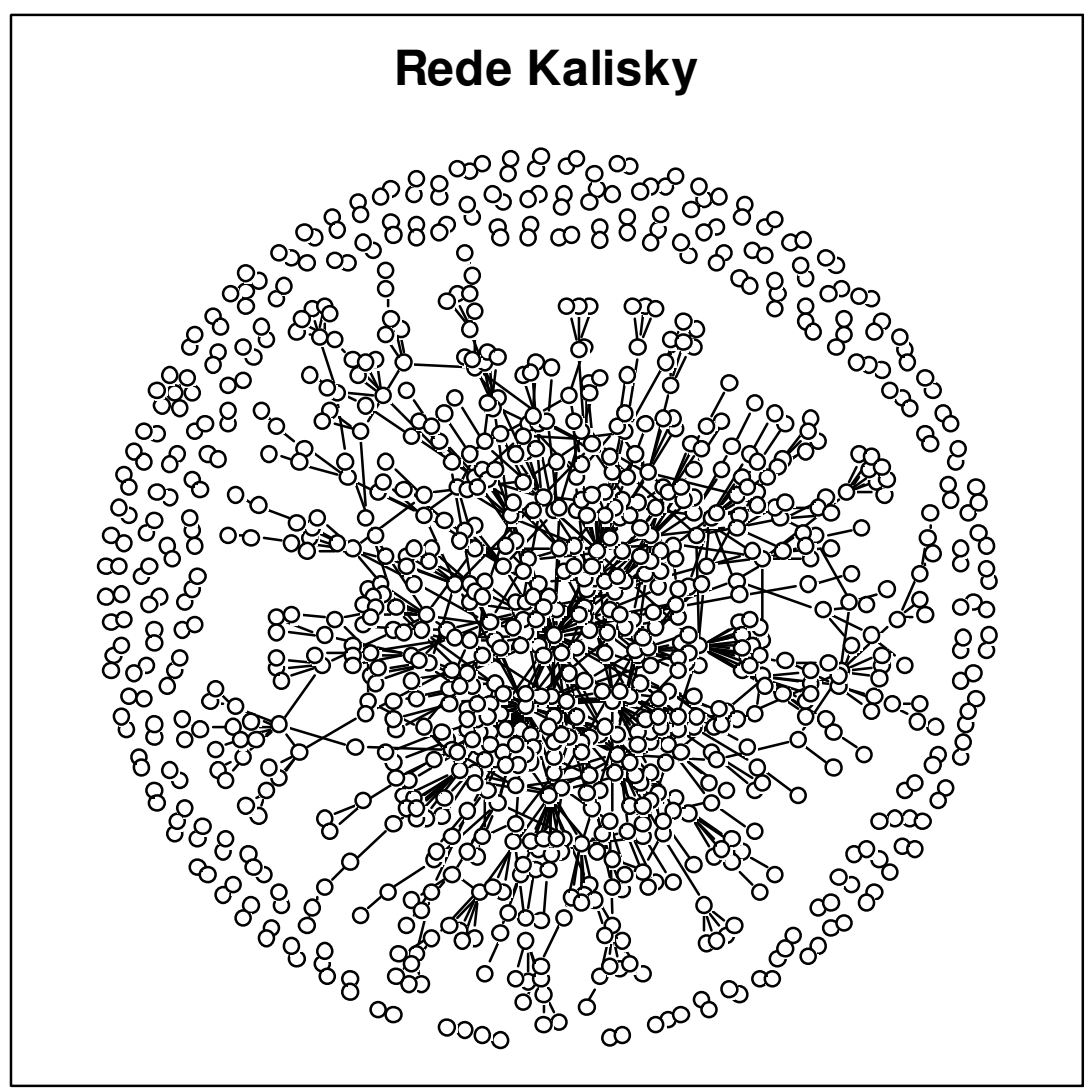

Figura 6 - Ilustração de uma rede que segue o modelo Kalisky, com $P(k)$ gerado a partir de uma rede Barabási-Albert com: vértices $=1000$ e $m=1$ 


\subsubsection{Modelo Molloy-Reed}

Mais detalhes deste algoritmo podem ser obtidos em (KALISKY; COHEN; HAVLIN, 2004), sendo representada na figura 7 uma rede que segue este modelo:

1. Cada vértice recebe um grau da distribuição.

2. A cada passo de tempo, conectamos aleatoriamente um par de vértices, considerando que a probabilidade de selecionar um vértice é diretamente proporcional ao número de arestas remanescentes.

3. Repete-se este processo até que não existam mais vértices com conexões a serem realizadas.

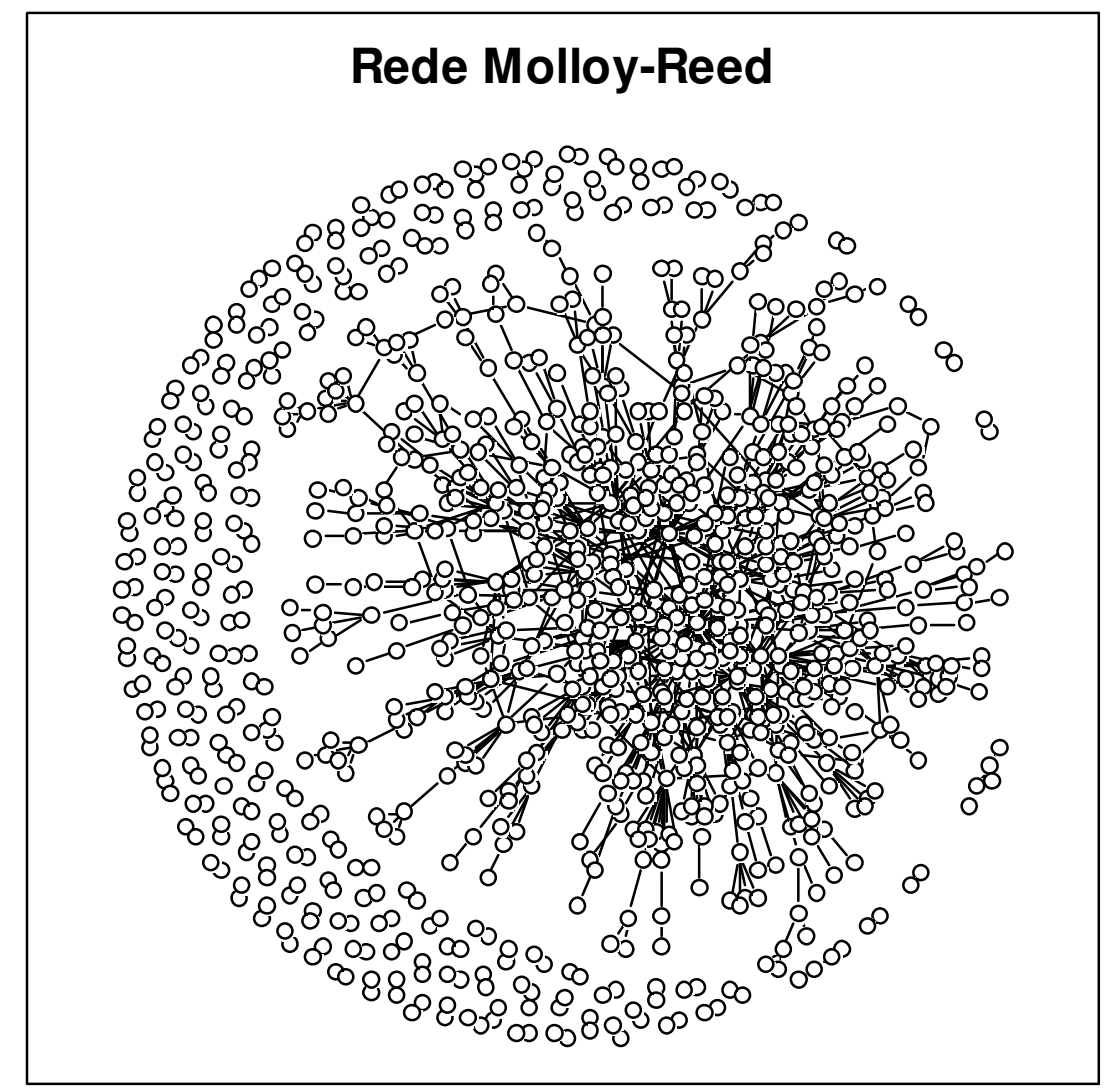

Figura 7 - Ilustração de uma rede que segue o modelo Molloy-Reed, com $P(k)$ gerado a partir de uma rede Barabási-Albert com: vértices $=1000$ e $m=1$ 
Neste projeto, para gerar as redes hipotéticas, primeiramente, geraram-se 10 redes seguindo o modelo Barabási-Albert, usando a função barabasi.game(n, m, directed) do pacote igraph, variando-se o número de vértices $(\mathrm{n}=1000,10000$ e 100000), o número de arestas de cada vértice $(m=1,2$ e 3$)$ e o parâmetro que define se a rede deve ser direcionada ou não (directed $=$ TRUE e FALSE). Então, usando a distribuição de graus destas redes BarabásiAlbert como entrada, a fim de garantir que todas as redes geradas seguiriam a mesma distribuição de graus e que as diferenças nas estruturas topológicas se devem ao modo como as redes foram construídas, gerou-se cada um dos outros modelos mencionados anteriormente. O quadro 1 resume a informação apresentada aqui.

\begin{tabular}{|c|c|c|c|c|}
\hline Modelos de redes & $\begin{array}{c}\text { Quantidade gerada } \\
\text { de cada modelo }\end{array}$ & $\mathbf{n}$ & $\mathbf{m}$ & directed \\
\hline \multirow{3}{*}{$\begin{array}{l}\text { Barabási-Albert, Molloy-Reed, } \\
\text { Kalisky, Método A e Método B }\end{array}$} & 10 & 1000 & \multirow{3}{*}{1} & \multirow{9}{*}{ FALSE } \\
\hline & 10 & 10000 & & \\
\hline & 10 & 100000 & & \\
\hline \multirow{3}{*}{$\begin{array}{l}\text { Barabási-Albert, Molloy-Reed, } \\
\text { Kalisky, Método A e Método B }\end{array}$} & 10 & 1000 & \multirow{3}{*}{2} & \\
\hline & 10 & 10000 & & \\
\hline & 10 & 100000 & & \\
\hline \multirow{3}{*}{$\begin{array}{l}\text { Barabási-Albert, Molloy-Reed, } \\
\text { Kalisky, Método A e Método B }\end{array}$} & 10 & 1000 & \multirow{3}{*}{3} & \\
\hline & 10 & 10000 & & \\
\hline & 10 & 100000 & & \\
\hline \multirow{3}{*}{$\begin{array}{l}\text { Barabási-Albert, Molloy-Reed, } \\
\text { Kalisky, Método A e Método B }\end{array}$} & 10 & 1000 & \multirow{3}{*}{1} & \multirow{9}{*}{ TRUE } \\
\hline & 10 & 10000 & & \\
\hline & 10 & 100000 & & \\
\hline \multirow{3}{*}{$\begin{array}{l}\text { Barabási-Albert, Molloy-Reed, } \\
\text { Kalisky, Método A e Método B }\end{array}$} & 10 & 1000 & \multirow{3}{*}{2} & \\
\hline & 10 & 10000 & & \\
\hline & 10 & 100000 & & \\
\hline \multirow{3}{*}{$\begin{array}{l}\text { Barabási-Albert, Molloy-Reed, } \\
\text { Kalisky, Método A e Método B }\end{array}$} & 10 & 1000 & \multirow{3}{*}{3} & \\
\hline & 10 & 10000 & & \\
\hline & 10 & 100000 & & \\
\hline
\end{tabular}

Quadro 1 - Tipos de redes geradas neste projeto. Com os valores adotados na função barabasi.game(n, m, directed) 


\subsection{REDE DO ESTADO DO MATO GROSSO}

Usando as informações sobre a movimentação de bovinos do banco de dados de guias de trânsito animal (GTA) do Instituto de Defesa Agropecuária do Estado do Mato Grosso INDEA/MT, referentes ao ano de 2007 (MATO GROSSO, 2008; NEGREIROS, 2010), foi possível construir uma rede oriunda de dados reais.

O banco de dados contém, entre outras informações, dados sobre a origem, o destino, a data e a finalidade de cada movimentação, além de um código único identificando cada fazenda. Assim sendo, após a verificação de possíveis inconsistências que poderiam existir neste banco e da retirada das movimentações que tinham como finalidade o abate dos bovinos, já que tais movimentações não contribuem para dinâmica da doença na rede (NATALE et al., 2009), restaram 86559 fazendas que realizaram 284834 movimentações de bovinos durante o ano de 2007. Como temos a informação sobre a data de cada movimentação, reproduzimos a rede de duas formas: considerando-a estática, para fins de comparação com as redes hipotéticas e considerando-a dinâmica, representando as movimentações realizadas neste período de forma mais realística. 


\subsection{MODELO DAS SIMULAÇÕES DA DINÂMICA DE ESPALHAMENTO}

A seguir, através da figura 8, apresentamos o modelo geral usado nas simulações para descrever a dinâmica de espalhamento de uma doença em uma rede de movimentação de animais, implementado usando o programa MATLAB.

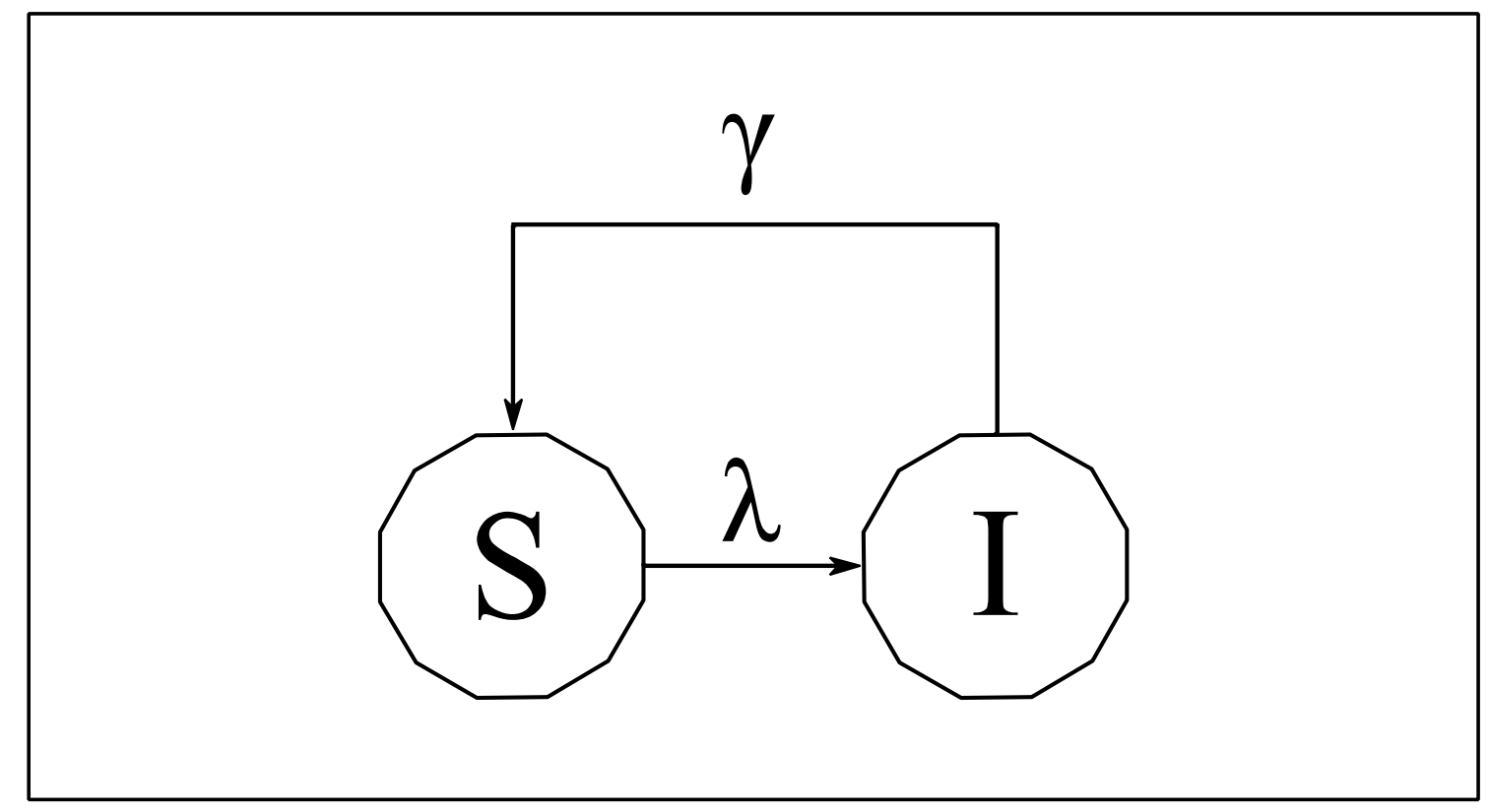

Figura 8 - Modelo de SIS (Suscetível - Infectado - Suscetível), onde $\lambda$ indica a probabilidade de infecção de uma fazenda e $\gamma$ é o período infeccioso

Onde:

$\mathbf{S}$, representa que a fazenda está suscetível a adquirir a infecção;

I, representa que a fazenda está infectada, isto é, possui animais infectados, podendo transmitir a infecção caso envie seus animais para uma fazenda suscetível;

$\lambda$, é a probabilidade de que uma fazenda suscetível, que receba animais de uma fazenda infectada, adquira a infecção. Para as simulações, assumimos: $\lambda=5 \%, 10 \%, 40 \%, 70 \%, 100 \%$;

$\gamma$, é a quantidade de unidades de tempo de simulação que uma fazenda permanecerá infectada. Modelado como: $\gamma \sim$ Uniforme $\left(t I_{\min }, t I_{\max }\right)$, onde $\boldsymbol{t \boldsymbol { I } _ { \operatorname { m i n } }}$ e $\boldsymbol{t \boldsymbol { I } _ { \operatorname { m a x } }}$ são, respectivamente, o tempo mínimo e o tempo máximo que uma fazenda poderá ficar no estado infectado; 
Variando os valores do parâmetro $\gamma$, simulamos o comportamento de doenças hipotéticas agudas e crônicas, para diferentes valores de $\lambda$, onde consideramos que uma vez que uma fazenda fica infectada, ela ficaria neste estado durante um tempo médio fixo (abordagem geralmente adotada quando não se tem uma informação mais precisa sobre a doença na população) ou que haveria uma variação neste tempo (representando de forma mais realista o processo de descoberta e de tratamento dos animais nas diferentes fazendas). O quadro 2 apresenta os valores adotados no parâmetro $\gamma$ para a realização das simulações.

\begin{tabular}{|c|c|c|c|c|}
\hline \multirow{2}{*}{ Modelos de espalhamento } & \multirow{2}{*}{ Doença hipotética } & \multirow{2}{*}{ Tempo } & \multicolumn{2}{|c|}{$\boldsymbol{\gamma}$} \\
\cline { 3 - 5 } & \multirow{2}{*}{ Crônica } & \multicolumn{1}{|c|}{ Fixo } & \multicolumn{2}{|c|}{$T+100$} \\
\hline \multirow{2}{*}{ SI } & \multirow{2}{*}{ Aguda } & Fixo & \multicolumn{2}{|c|}{30} \\
\hline \multirow{2}{*}{ SIS } & \multirow{3}{*}{ Crônica } & Fariável & 15 & 45 \\
\cline { 3 - 5 } & & Farixo & \multicolumn{2}{|c|}{360} \\
\cline { 3 - 5 } & & Variável 1 & 330 & 390 \\
\cline { 3 - 5 } & \multirow{2}{*}{ Cróvel 2 } & 180 & 540 \\
\hline
\end{tabular}

Quadro 2 - Valores adotados no parâmetro $\gamma$ para a realização dos diferentes modelos de espalhamento, onde $T$ é o tempo total da simulação

As simulações foram realizadas iniciando-se com $50 \%$ da rede infectada, pois em um trabalho anterior (OSSADA et al., 2010) verificamos que a variação no número inicial de vértices infectados causa apenas uma mudança nas oscilações iniciais da média da prevalência. Adotamos um tempo total de simulação $(\boldsymbol{T})$ de 1000 unidades de tempo, para as redes hipotéticas, e de 1095 unidades de tempo para a rede real do Estado do Mato Grosso, onde no caso da versão dinâmica desta rede, estas unidades de tempo representam os dias do ano e supomos, para as simulações, que o comportamento da rede se manteria o mesmo durante 3 anos, permitindo assim uma melhor comparação com as simulações nas redes hipotéticas.

Para cada modelo de espalhamento, a metodologia usada nas simulações foi de (a) realizar 100 simulações de espalhamento para cada rede, calculando a prevalência da doença nestas e então (b) calcular a média da prevalência destas simulações e então (c) agrupar essas simulações pelo modelo de rede e (d) calcular a média da prevalência deste (A Figura 9 apresenta as informações aqui descritas de forma gráfica, usando as simulações SIS de doença aguda com tempo variável na rede Kalisky direcionada como exemplo). 
Rede direcionada, Kalisky, $m=3$, vértices $=1000, \lambda=0.7$ SIS Aguda Variável

(a) 100 Simulações na rede Kalisky 1

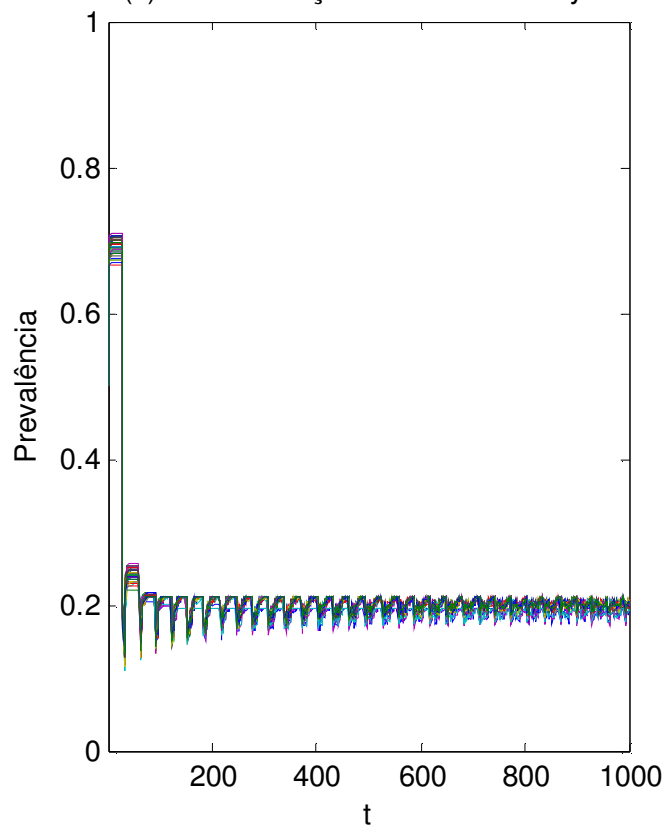

(c) Médias das simulações das 10 redes Kalisky

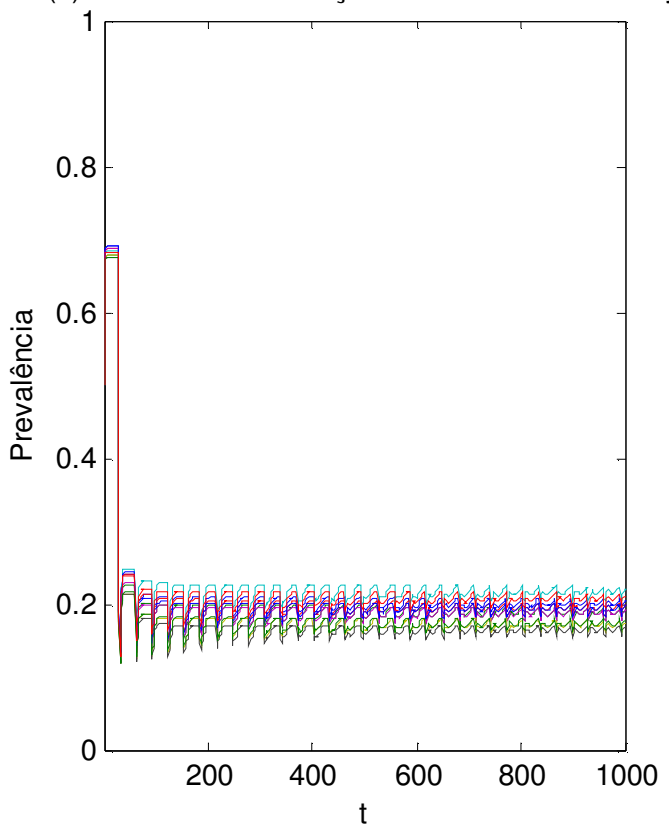

(b) Média das simulações na rede Kalisky 1

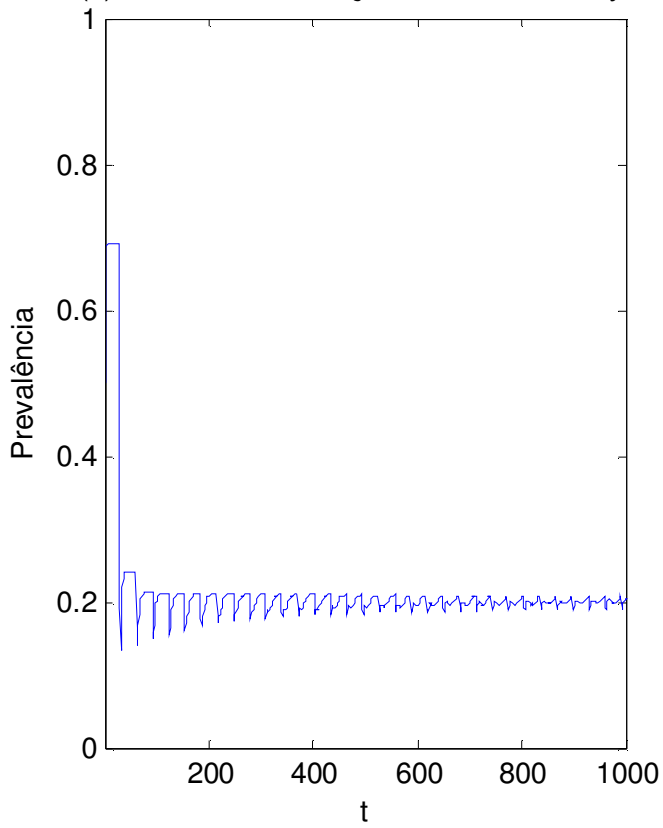

(d) Média da rede Kalisky

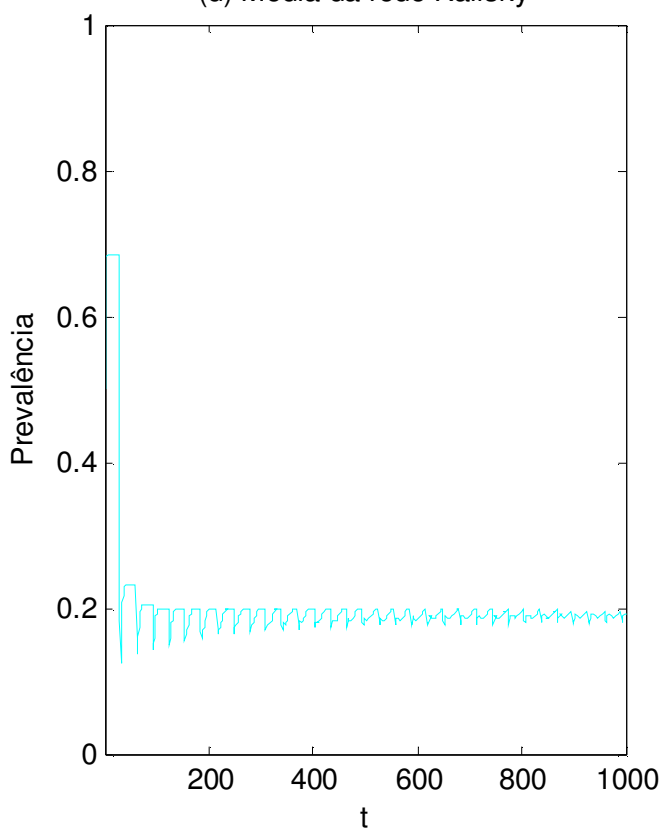

Figura 9 - Ilustração da metodologia usada nas simulações de espalhamento de doenças. (a) e (b) Simulações realizadas em 1 rede Kalisky (Kalisky 1); (c) e (d) Simulações nas 10 redes Kalisky geradas no projeto 


\section{RESULTADOS}

A seguir, devido à grande quantidade de simulações realizadas e conseqüentemente de resultados obtidos, omitimos alguns destes resultados, apresentando apenas aqueles que foram considerados os mais representativos deste projeto.

\subsection{SIMULAÇÕES NAS REDES NÃO DIRECIONADAS}

Nestas redes, observou-se que a doença se espalha independentemente do valor de $\lambda$ adotado, e que quando há o aumento de $\lambda$, há o aumento da prevalência da infecção nas redes (A Figura 10 ilustra essa informação utilizando a rede não direcionada Método B como exemplo).

Observamos também que o acréscimo de vértices não apresenta grande influência sobre a prevalência da infecção nas redes, já que a prevalência tende a se estabilizar num mesmo patamar apesar do aumento do número de vértices (A Figura 11 ilustra essa informação utilizando a rede não direcionada Método A como exemplo).

Vemos também que à medida que se aumenta o número de arestas de cada vértice, há um aumento na prevalência da infecção. E que a rede Método B, apresenta os menores valores de prevalência em todas as simulações (Figuras 12, 13 e 14).

Dentre os resultados, o que mais chama a atenção é que quando $m=1$ existe uma grande diferença entre os patamares de equilíbrio de prevalência em cada rede, mas à medida que o $m$ aumenta, as redes tendem a apresentar valores muito próximos de equilíbrio (Figuras 12, 13 e 14). 


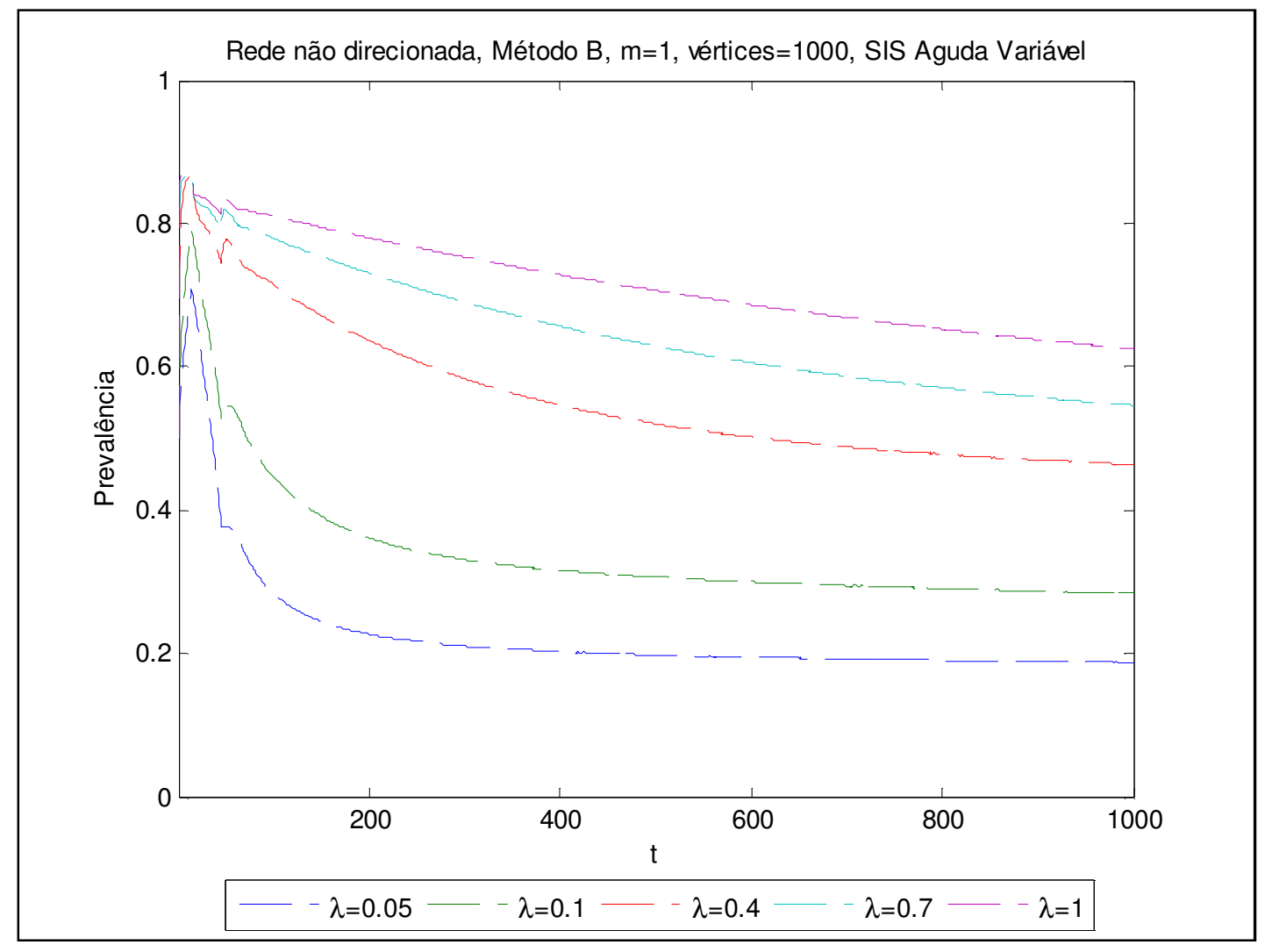

Figura 10 - Efeito do aumento do valor de $\lambda$ numa rede não direcionada

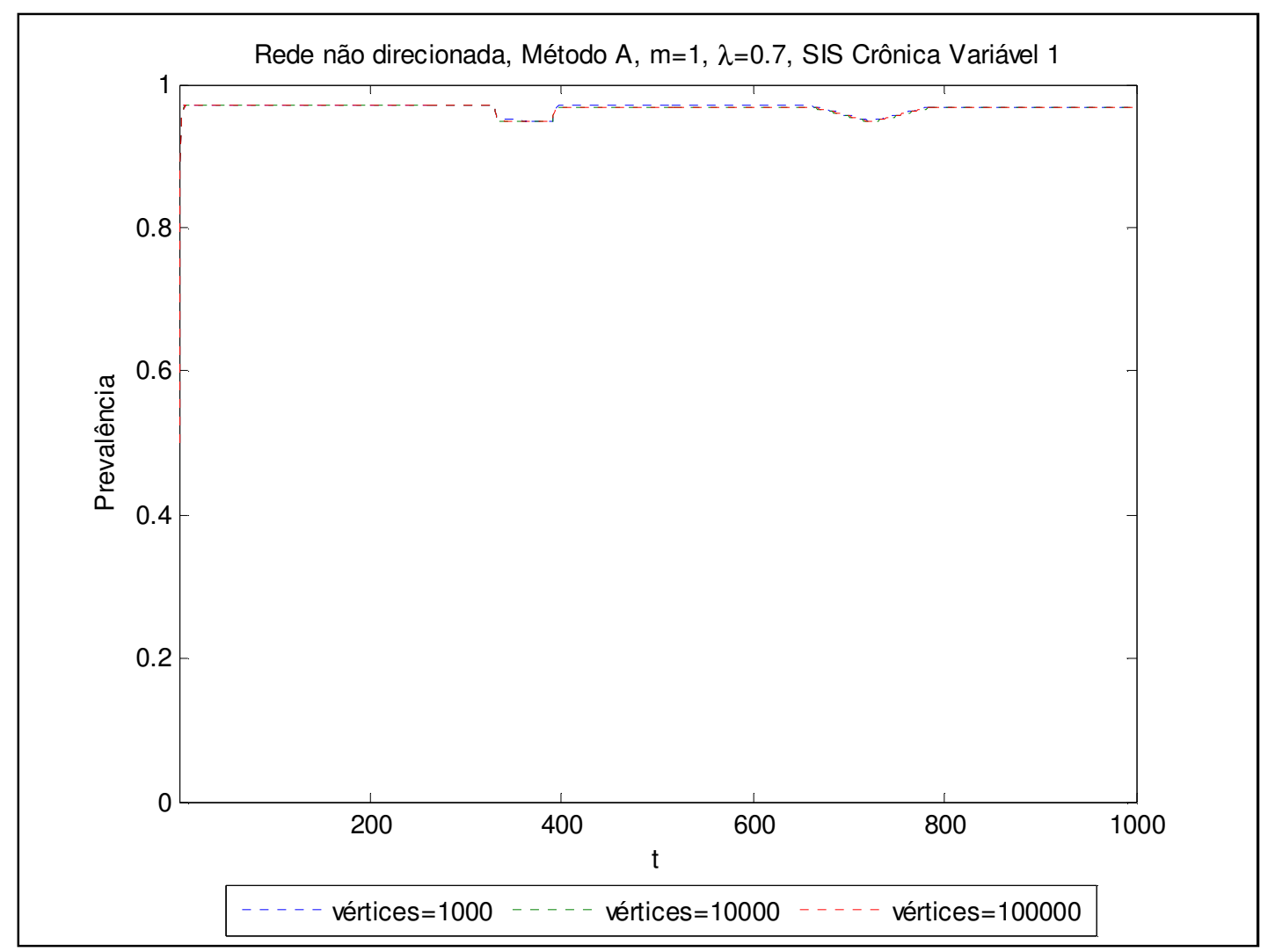

Figura 11 - Efeito do aumento do número de vértices numa rede não direcionada 
Redes não direcionadas, $m=1$, vértices $=1000, \lambda=0.70$
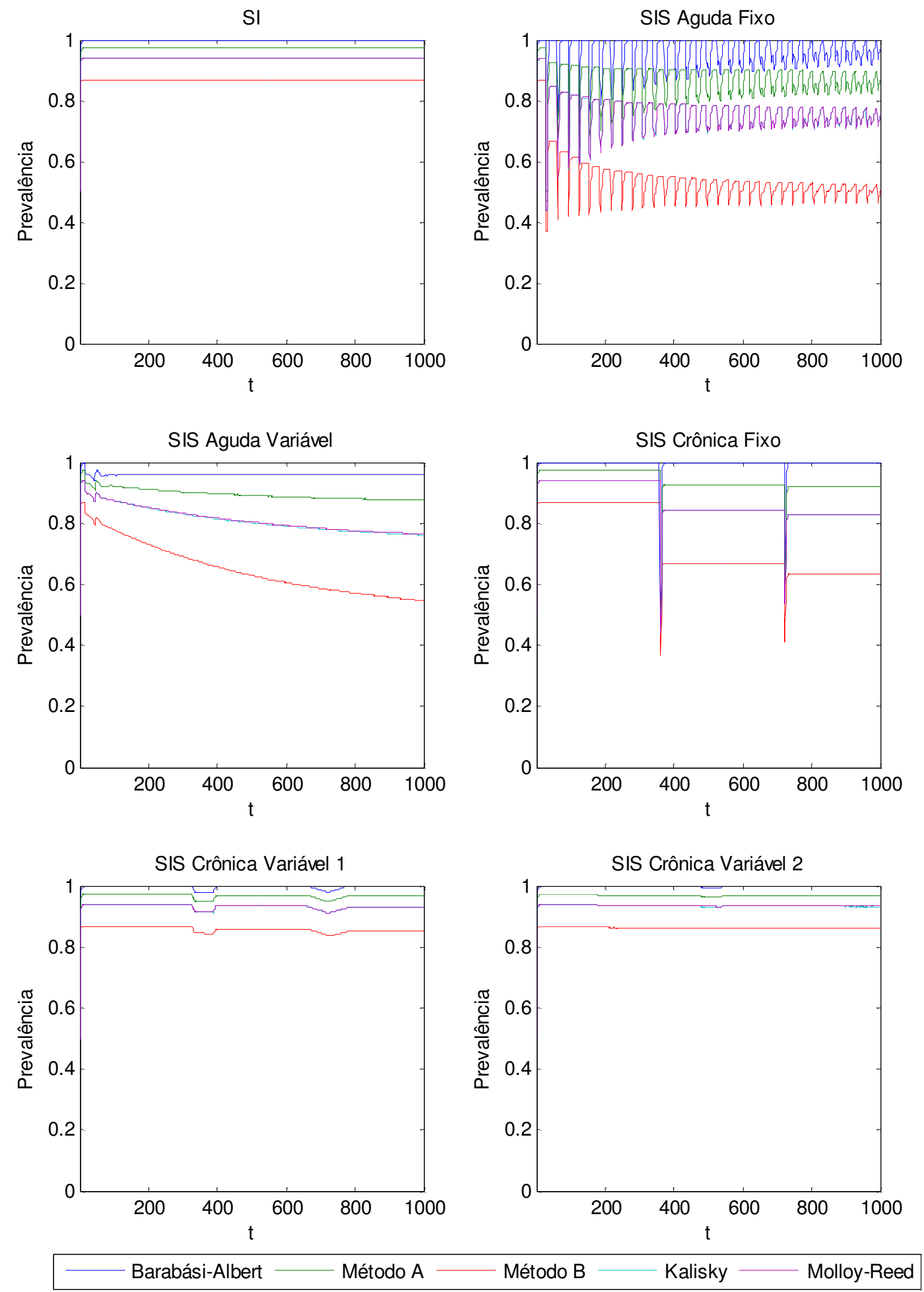

Figura 12 - Simulações de espalhamento nas redes não direcionadas quando $m=1$ 
Redes não direcionadas, $m=2$, vértices $=1000, \lambda=0.70$
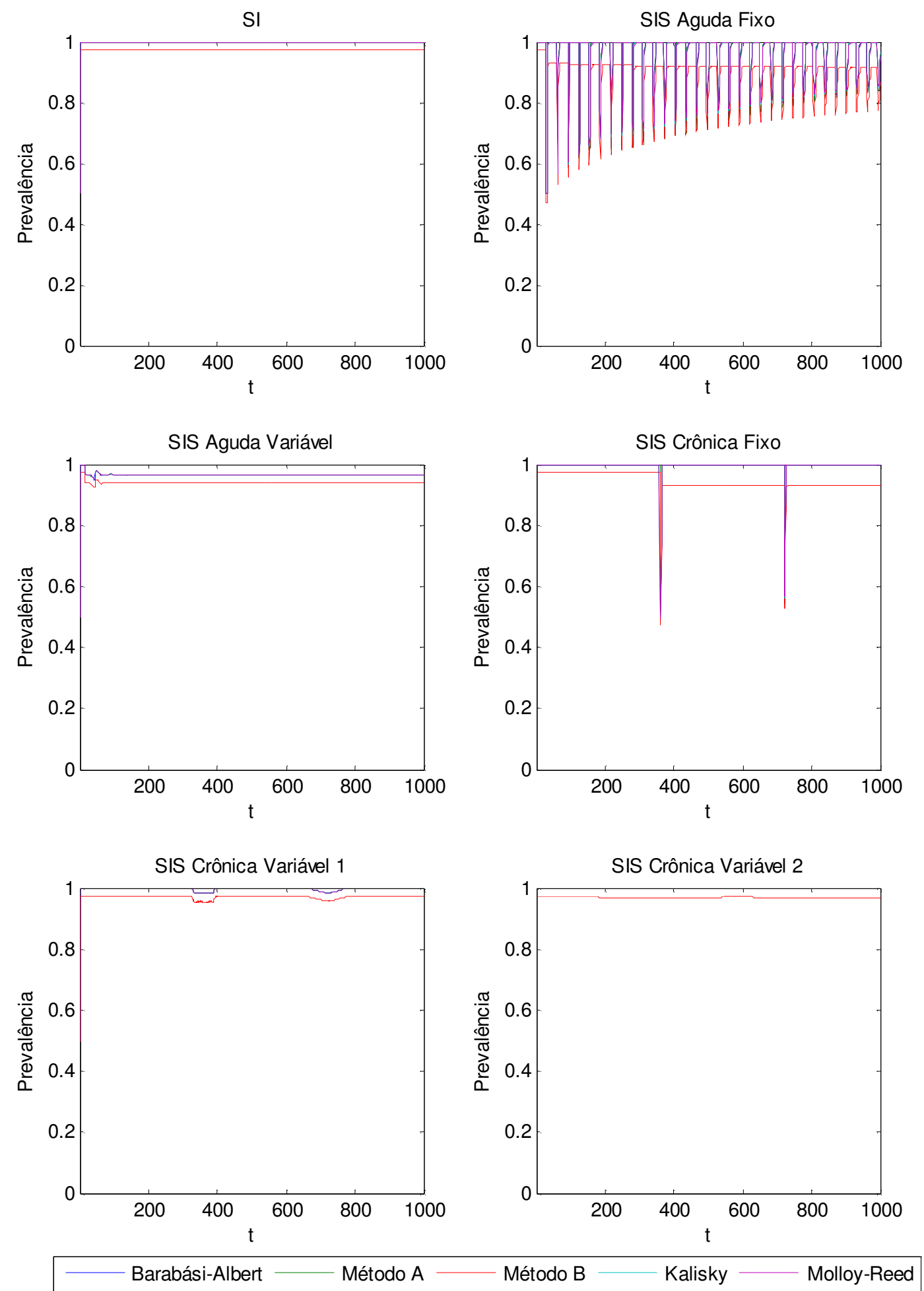

Figura 13 - Simulações de espalhamento nas redes não direcionadas quando $m=2$ 
Redes não direcionadas, $m=3$, vértices $=1000, \lambda=0.70$

SI
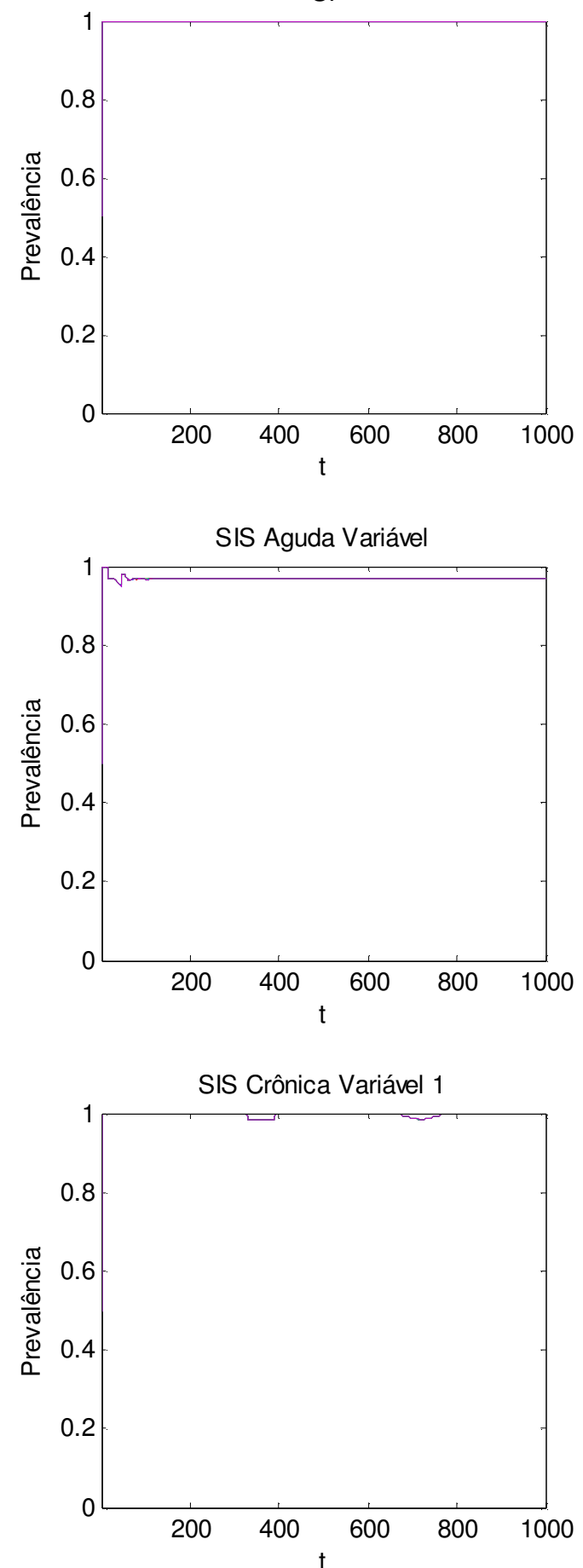

SIS Aguda Fixo
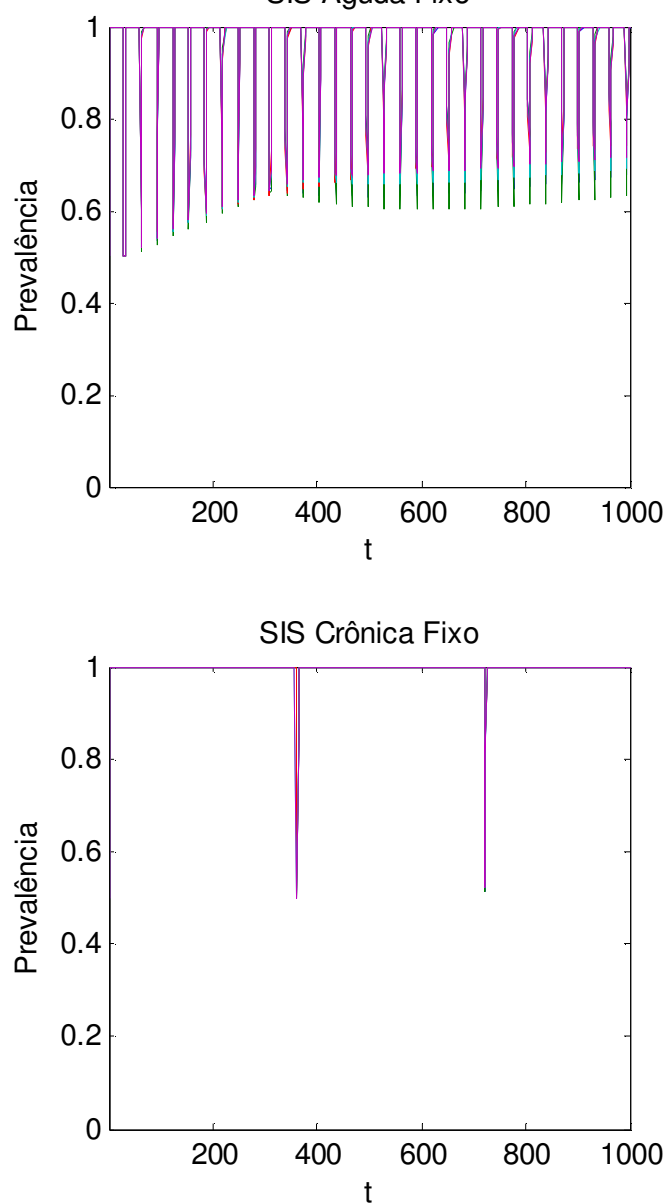

SIS Crônica Variável 2

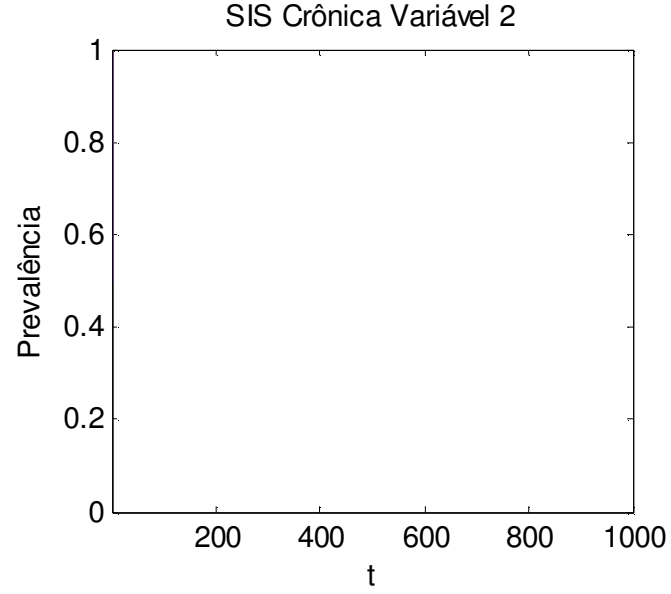

Barabási-Albert — Método A

Método B

Kalisky

Molloy-Reed

Figura 14 - Simulações de espalhamento nas redes não direcionadas quando $m=3$ 


\subsection{SIMULAÇÕES NAS REDES DIRECIONADAS}

Nestas redes, observou-se que, à exceção das simulações de infecções agudas, a doença se espalha independentemente do valor de $\lambda$ adotado, e assim como nas redes não direcionadas, quando há o aumento de $\lambda$, há o aumento da prevalência da infecção nas redes (A Figura 15 ilustra essa informação utilizando a rede direcionada Barabási-Albert como exemplo).

Observamos também que o acréscimo de vértices não apresenta grande influência sobre a prevalência da infecção nas redes, já que a prevalência tende a se estabilizar num mesmo patamar apesar do aumento do número de vértices (A Figura 16 ilustra essa informação utilizando a rede direcionada Método A como exemplo).

Vemos também que à medida que se aumenta o número de arestas de cada vértice, há um aumento na prevalência da infecção. E que a rede Barabási-Albert, apresenta os menores valores de prevalência em todas as simulações, nunca se aproximando dos resultados observados nas outras redes (Figuras 17, 18 e 19). E que quando $m=3$, a prevalência nas redes Kalisky tendem a equilibrar-se num patamar um pouco acima das outras redes (Figuras 17, 18 e 19). 
Rede direcionada, Barabási-Albert, $m=1$, vértices $=1000$
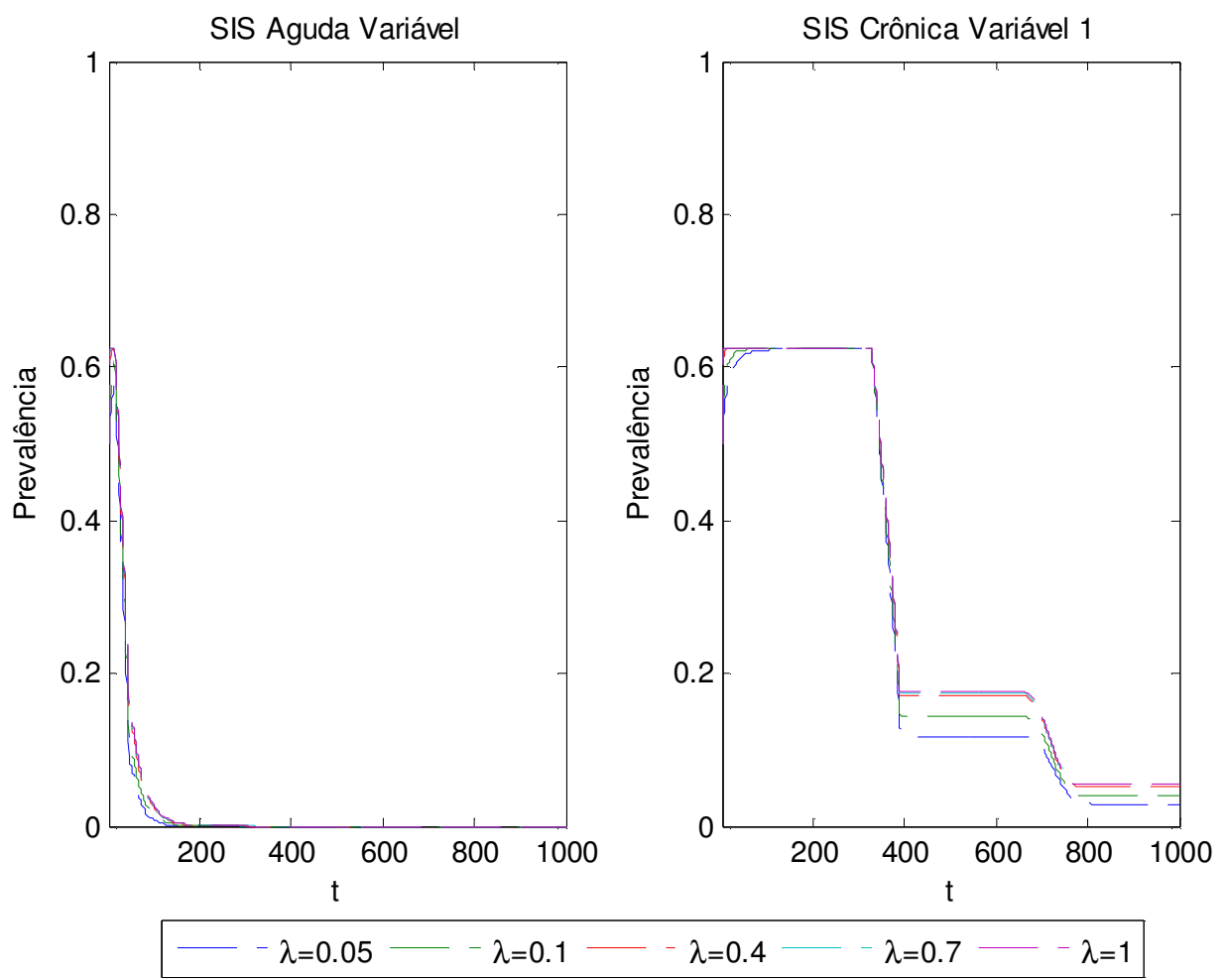

Figura 15 - Efeito do aumento do valor de $\lambda$ numa rede direcionada

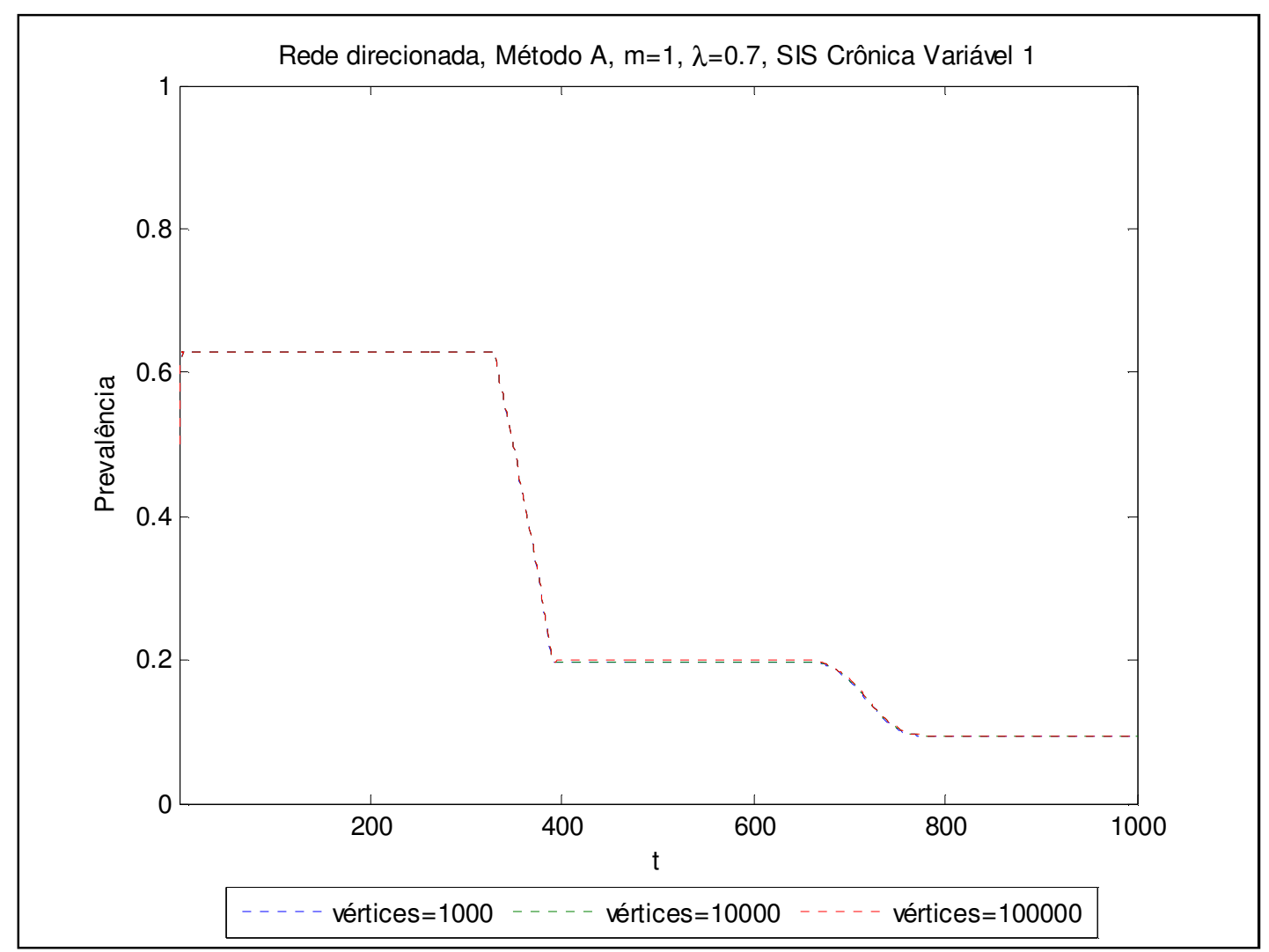

Figura 16 - Efeito do aumento do número de vértices numa rede direcionada 
Redes direcionadas, $m=1$, vértices $=1000, \lambda=0.70$

SI

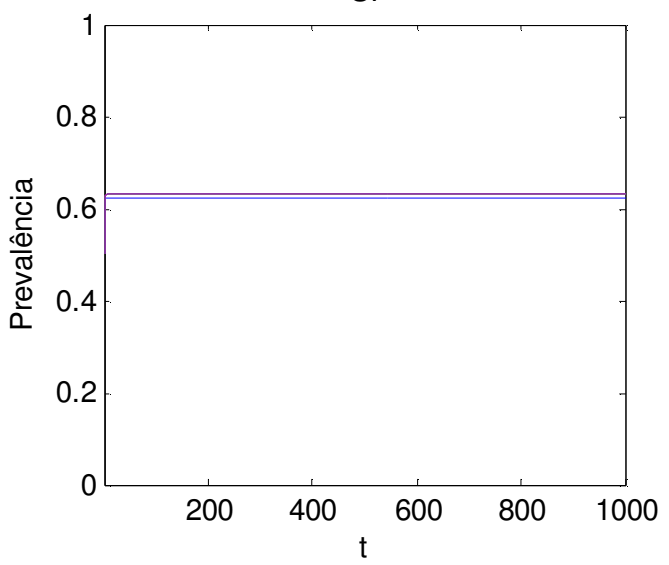

SIS Aguda Variável
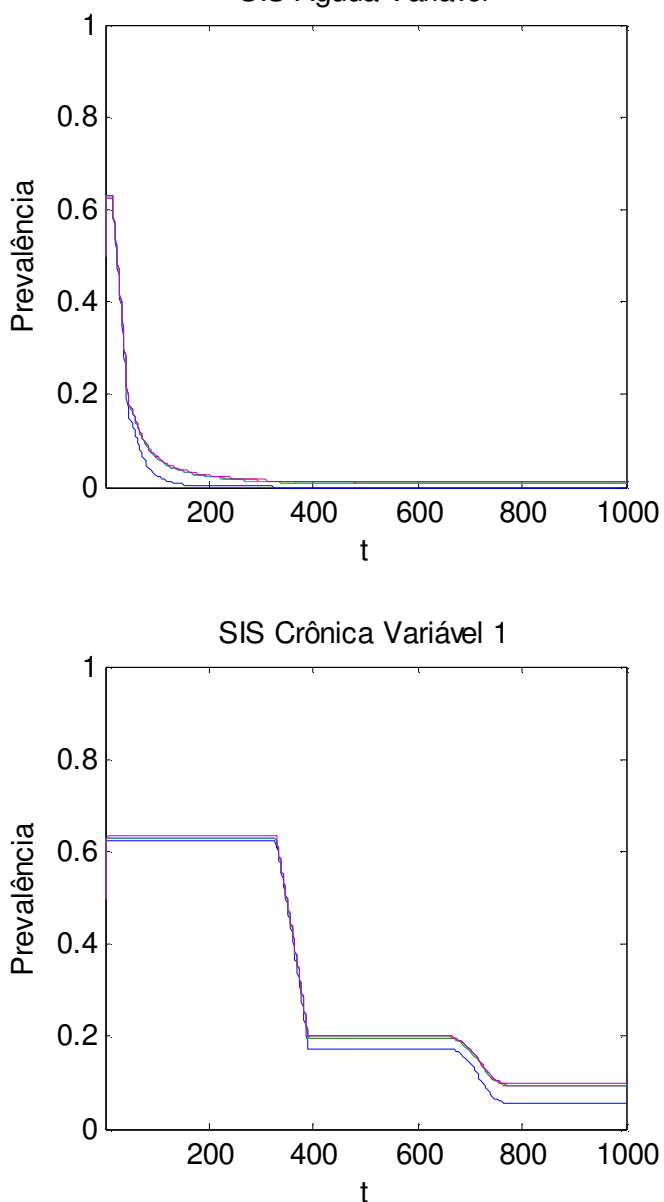

SIS Aguda Fixo

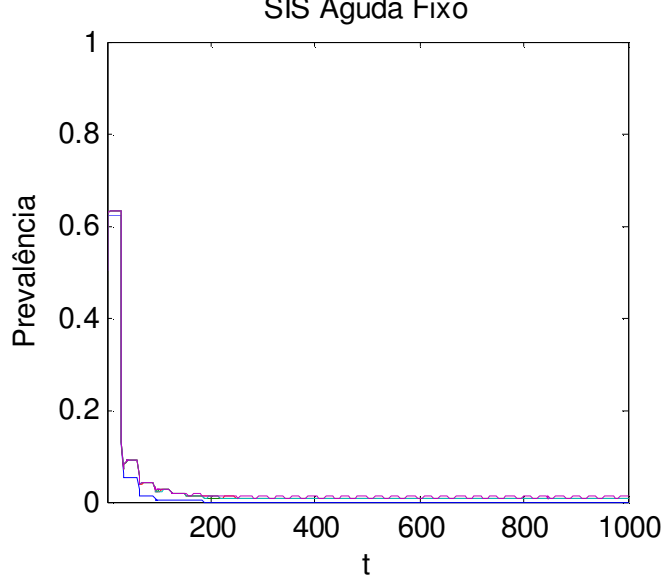

SIS Crônica Fixo

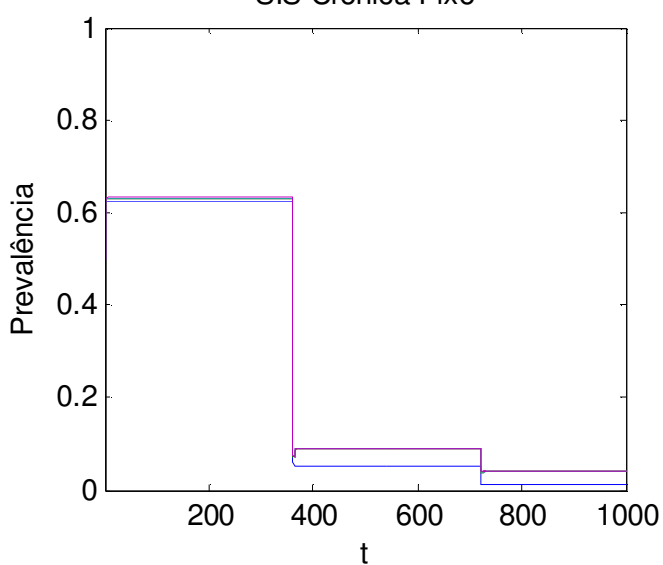

SIS Crônica Variável 2

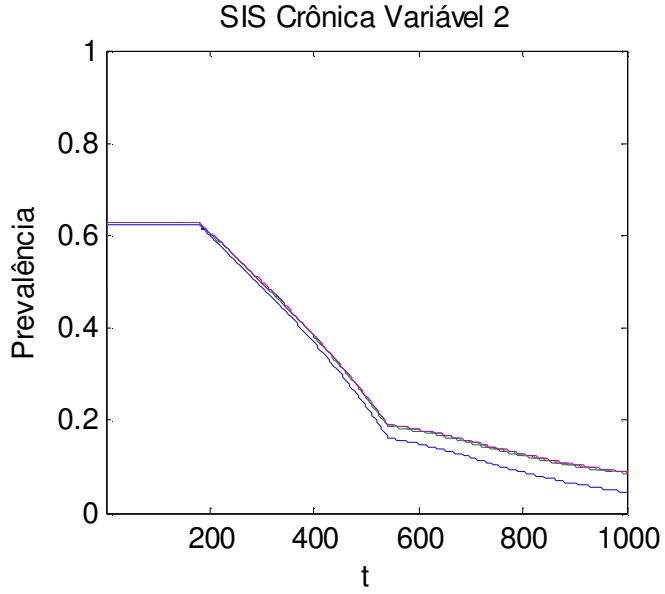

Barabási-Albert — Método A

Método B

Kalisky Molloy-Reed

Figura 17 - Simulações de espalhamento nas redes direcionadas quando $m=1$ 
Redes direcionadas, $m=2$, vértices $=1000, \lambda=0.70$

SI

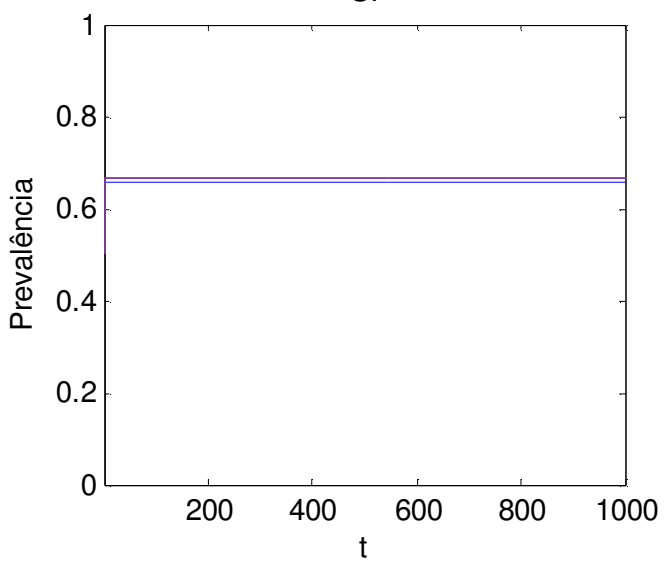

SIS Aguda Variável
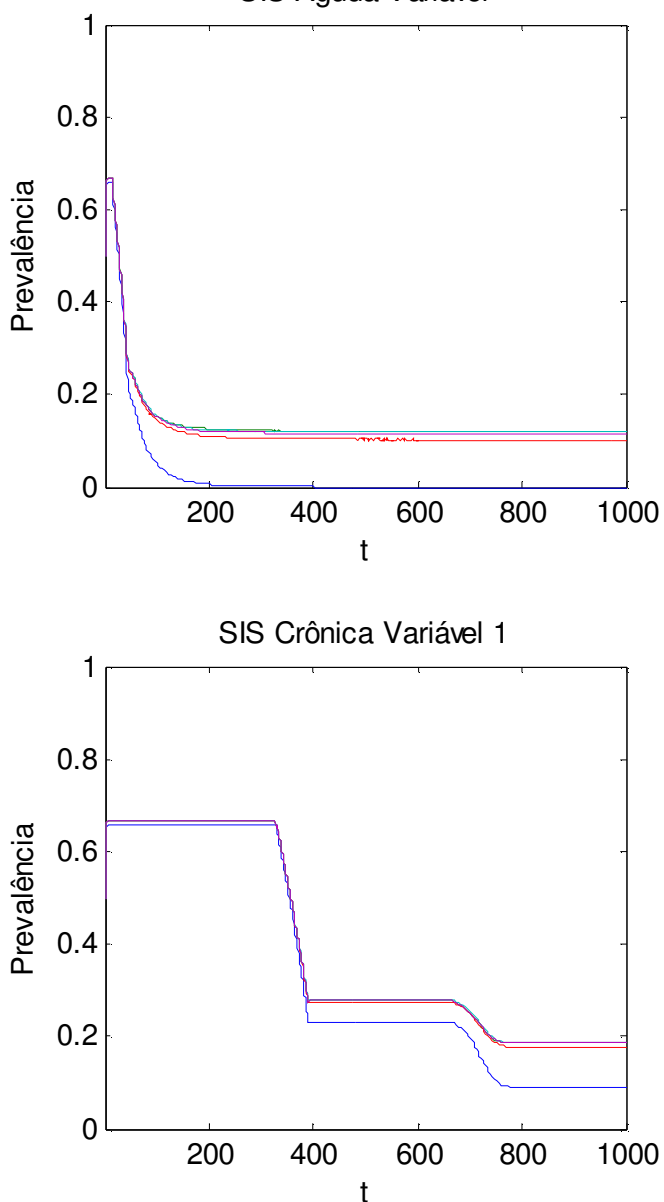

SIS Aguda Fixo

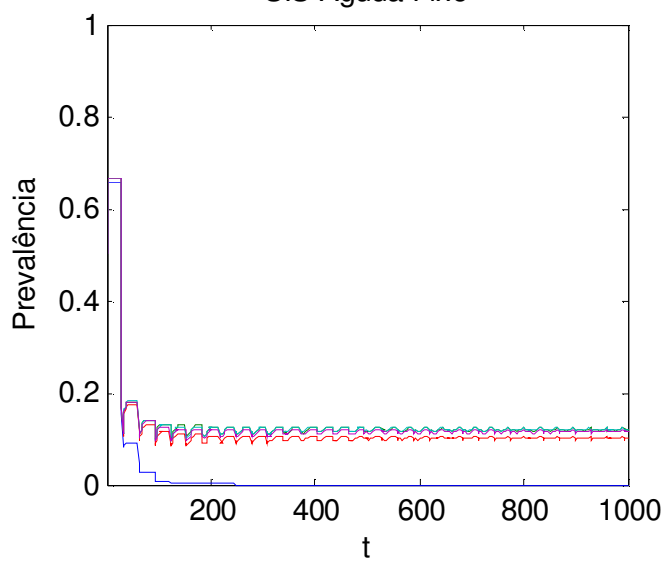

SIS Crônica Fixo

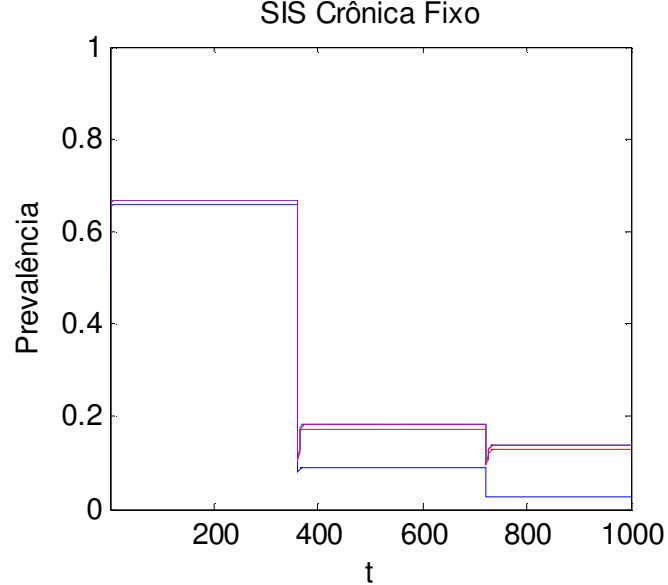

SIS Crônica Variável 2

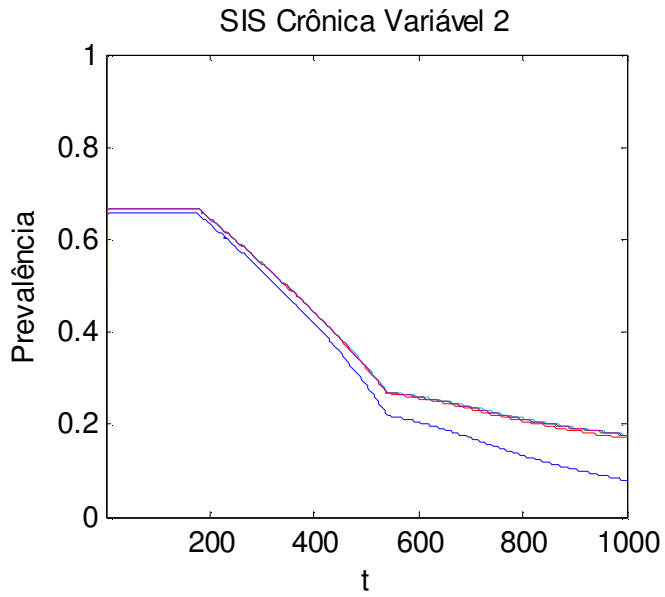

Barabási-Albert — Método A

Método B

Kalisky Molloy-Reed

Figura 18 - Simulações de espalhamento nas redes direcionadas quando $m=2$ 
Redes direcionadas, $m=3$, vértices $=1000, \lambda=0.70$

SI

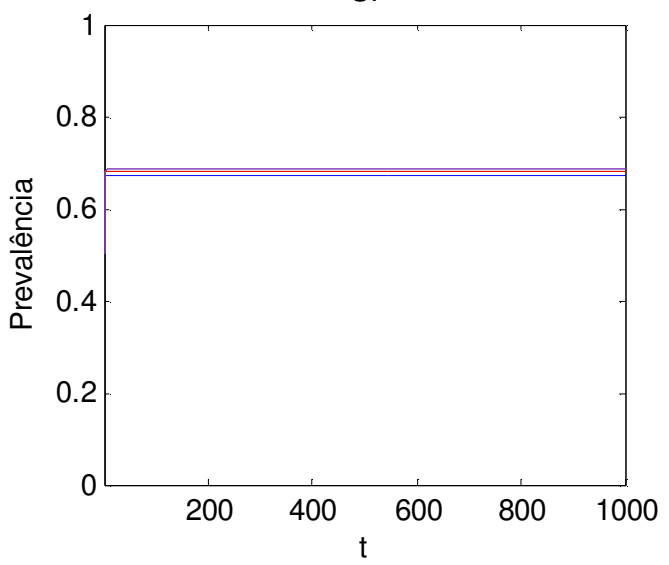

SIS Aguda Variável

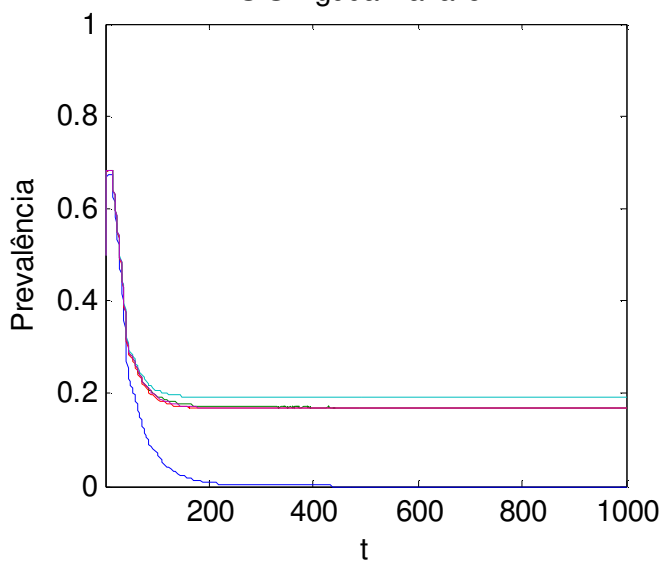

SIS Crônica Variável 1

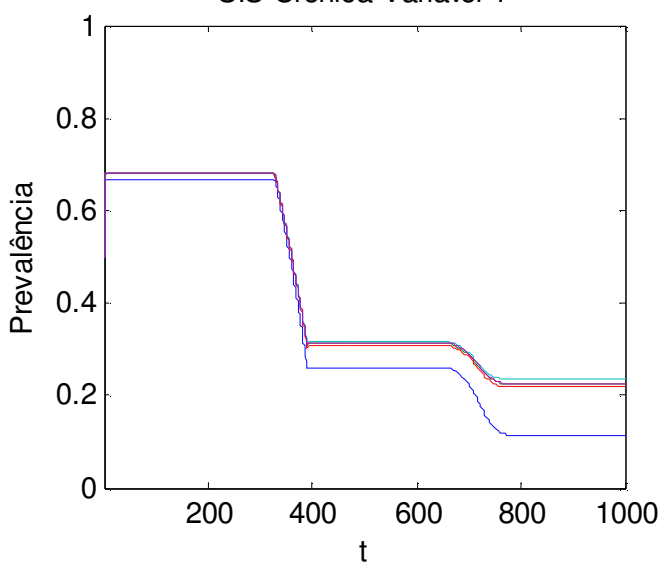

SIS Aguda Fixo

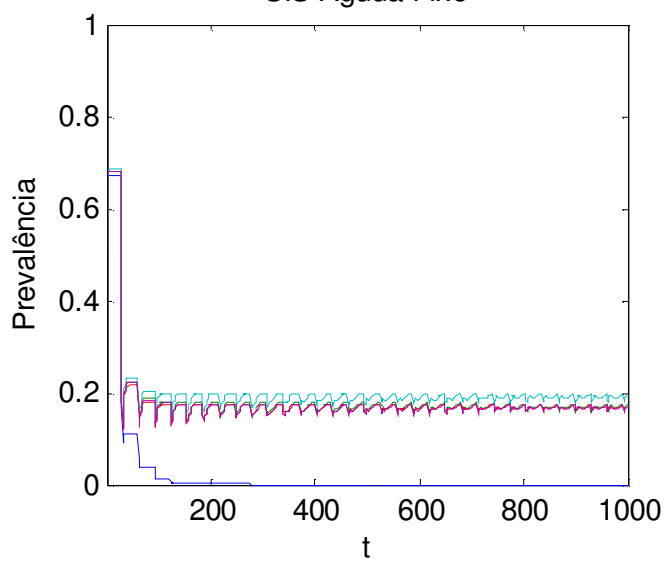

SIS Crônica Fixo

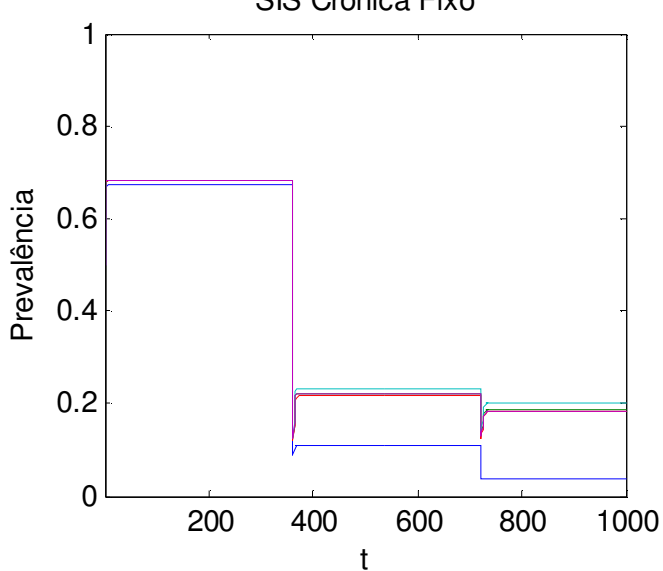

SIS Crônica Variável 2

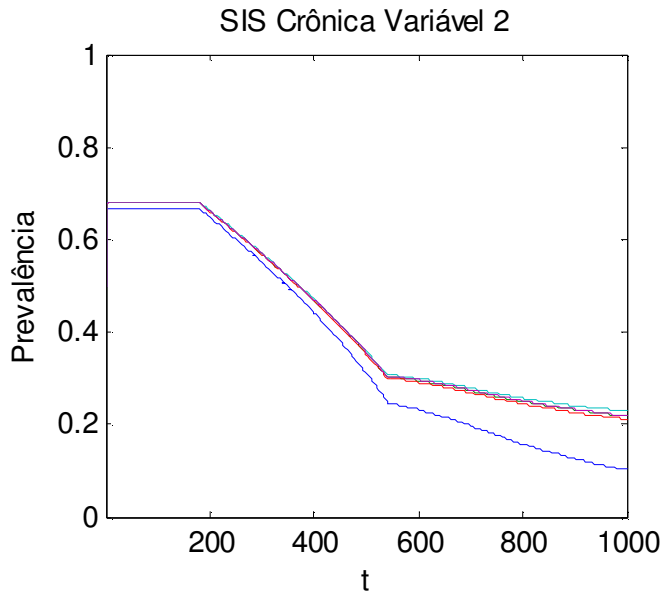

Barabási-Albert — Método A

Método B

Kalisky

Molloy-Reed

Figura 19 - Simulações de espalhamento nas redes direcionadas quando $m=3$ 


\subsection{SIMULAÇÕES NA REDE DO MATO GROSSO}

No caso da versão estática, observou-se que a doença se espalha independentemente do valor de $\lambda$ adotado, e assim como nas redes hipotéticas, quando há o aumento de $\lambda$, há o aumento da prevalência da infecção nas redes (Figura 20).

Já no caso da versão dinâmica, observou-se que, à exceção das simulações de infecções agudas, e das infecções crônicas com tempo fixo e tempo variável para $\lambda=5 \%$, a doença se espalha independentemente do valor de $\lambda$ adotado, e assim como nas redes hipotéticas, quando há o aumento de $\lambda$, há o aumento da prevalência da infecção nas redes (Figura 21).

Destacamos também que os valores de equilíbrio de prevalência das redes hipotéticas não direcionadas são superiores aos da rede real estática, que por sua vez são superiores aos das redes hipotéticas direcionadas, que são superiores aos da rede real dinâmica. 
Rede estática do Mato Grosso, vértices $=86559, \lambda=0.70$
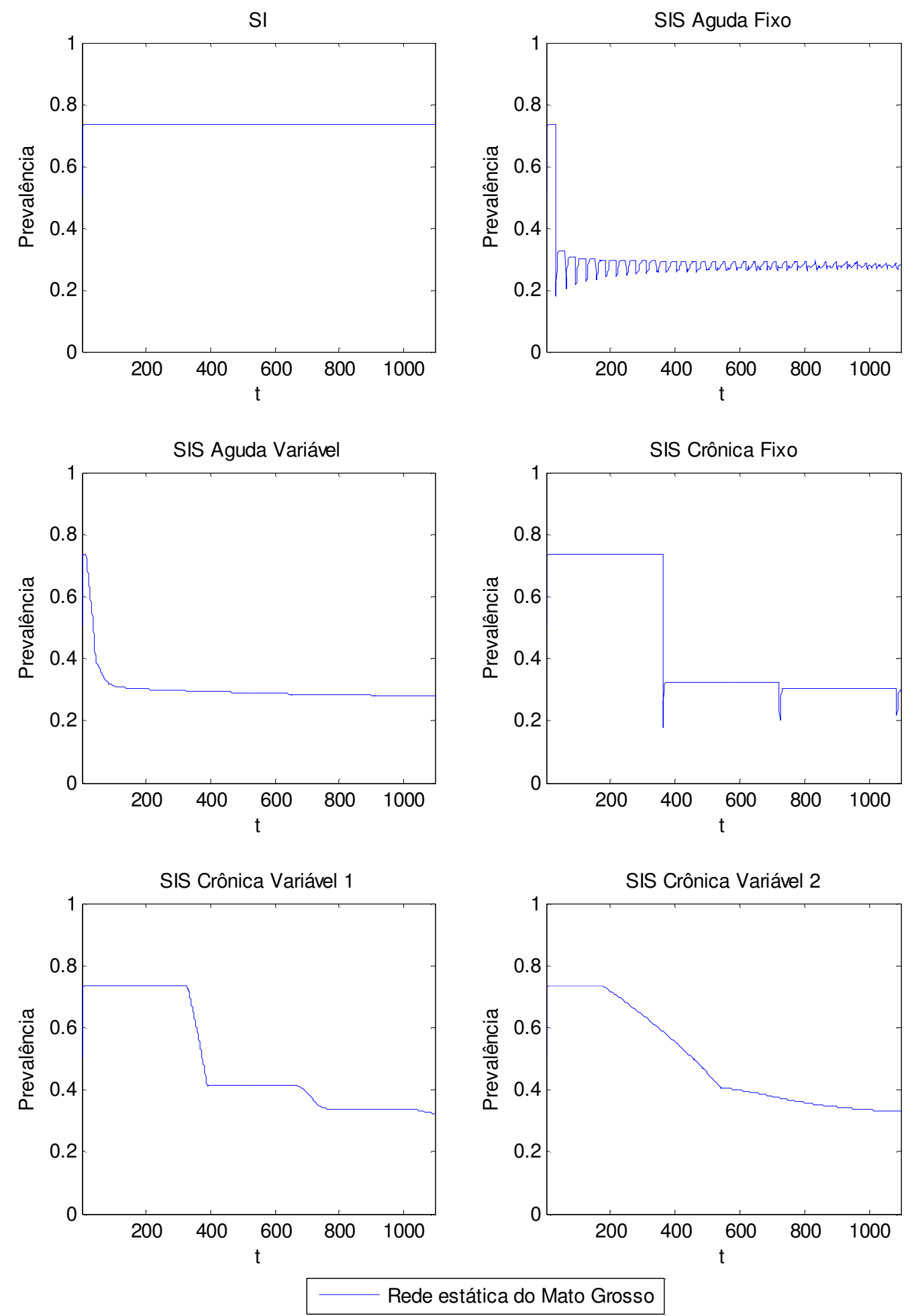

Figura 20 - Simulações de espalhamento na rede estática do Mato Grosso 
Rede dinâmica do Mato Grosso, vértices $=86559, \lambda=0.70$
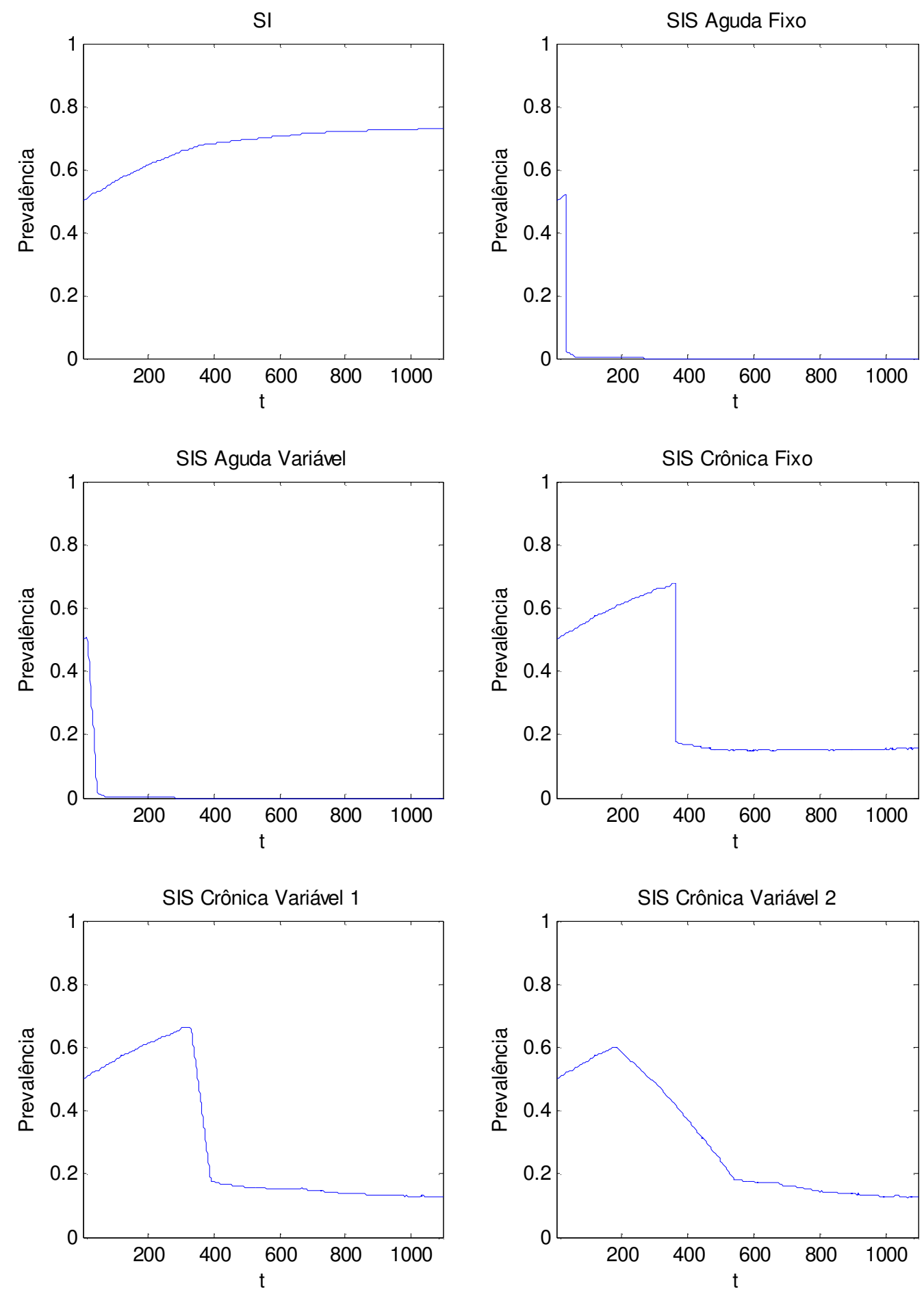

Rede dinâmica do Mato Grosso

Figura 21 - Simulações de espalhamento na rede dinâmica do Mato Grosso 


\section{DISCUSSÃO}

Analisando os resultados obtidos nas simulações, temos que, como esperado, a variação no tamanho das redes hipotéticas pouco influenciou na prevalência das doenças teóricas, o que é consistente com as características das redes complexas livres de escala, como observado em Pastor-Satorras e Vespignani (2001). Com relação à variação do número de arestas de cada vértice, podemos dizer que o aumento da prevalência observado também era esperado, pois sabe-se que o acréscimo de arestas aumenta a conectividade da rede, possibilitando que a doença se espalhe com mais facilidade nesta. Sobre o efeito de considerar a rede como não direcionada ou direcionada, temos que, como esperado, no caso não direcionado as doenças tendem a permanecer na rede independentemente do modelo de espalhamento usado e do valor de $\lambda$ considerado (PASTOR-SATORRAS; VESPIGNANI, 2001), enquanto que, no caso direcionado, devido à limitação imposta pela direção da movimentação, quando $m=1$ as doenças agudas tendem a desaparecer destas redes.

Nas simulações, os patamares de estabilidade da prevalência observados resultam da recuperação simultânea de um conjunto de vértices. No caso de tempo de infecção fixo, o conjunto de vértices que se recupera simultaneamente é maior do que no caso de tempo de infecção variável, já que neste último, devido à variabilidade da duração da doença, formamse subconjuntos que vão se recuperar em momentos diferentes, enquanto que no caso de tempo fixo, os vértices de todo o conjunto deixarão o estado infectado no mesmo momento.

Examinando os resultados das simulações em cada modelo de rede, temos que a rede não direcionada Método B apresenta os menores valores de prevalência, sendo uma causa plausível para isto o modo como esta rede é formada, onde tem-se uma grande quantidade de vértices que não estão ligados à porção/componente mais conectada da rede. Já, no caso da rede direcionada Barabási-Albert, a baixa prevalência observada também se deve ao modo como esta rede é formada, pois ela é composta por diversos vértices que só enviam animais e por poucos vértices que recebem muitos animais e enviam muito pouco, dificultando assim o espalhamento de doenças.

Outro fato importante a se mencionar é que no caso das simulações de espalhamento de doenças crônicas nas redes direcionadas, quando $m=1$ o uso de um tempo médio fixo leva a doença a se estabilizar num patamar muito mais baixo do que o observado nos casos com tempo variável. 
Em relação à rede do Mato Grosso de 2007, vemos que as prevalências nela observadas (na versão estática) são maiores do que das redes direcionadas, o que pode indicar que a estrutura topológica da rede do Mato Grosso favorece o espalhamento de doenças. E comparando as prevalências entre as versões estática e dinâmica, como esperado, temos que as prevalências na versão estática são maiores que na versão dinâmica, já que nesta temos a influência da dinâmica das conexões da rede (que apresenta alguns períodos em que não há comercialização dos animais) sobre a dinâmica de espalhamento (VERNON; KEELING, 2009), constatado também através do desaparecimento das doenças agudas hipotéticas e da demora para a doença atingir o equilíbrio nas simulações SI. 


\section{CONCLUSÕES}

Com o uso da metodologia de redes, é possível analisar de forma mais clara os efeitos que a heterogeneidade das relações entre as fazendas têm sobre o espalhamento de doenças infecciosas, uma vez que vimos diferentes dinâmicas de espalhamento de doenças infecciosas nas redes geradas com o mesmo $\mathrm{P}(\mathrm{k})$ mas com estruturas topológicas distintas.

Através da análise da rede do Estado do Mato Grosso, vimos que quando tratando de dados reais, é interessante realizar simulações de espalhamento considerando esta rede como sendo estática e dinâmica, a fim de tentar entender melhor os efeitos da dinâmica da rede sobre a dinâmica da doença. Além disso, considerando que o aumento no número de arestas leva a um aumento da prevalência da doença na rede, temos indicativos de que a intensificação do comércio entre as fazendas pode favorecer o espalhamento de doenças. E que, em casos de emergência sanitária, a restrição do trânsito animal pode contribuir para controlar a doença.

Acreditamos que o objetivo de modelar a dinâmica do espalhamento de doenças infecciosas em redes de movimentação de animais foi atingido, uma vez que realizamos uma série de simulações de espalhamento em redes hipotéticas e depois aplicamos esta metodologia na rede de movimentação de bovinos do Estado do Mato Grosso de 2007.

Esperamos que os resultados aqui apresentados possam auxiliar nas atividades dos órgãos responsáveis pela vigilância epidemiológica e incentivem outros Estados a seguirem o exemplo do Estado do Mato Grosso, a construírem bancos de dados que possam ser analisados utilizando a metodologia de redes. 


\section{REFERÊNCIAS}

BARABÁSI, A-L.; ALBERT, R. Emergence of scaling in random networks. Science, v. 286, n. 5439, p. 509-512, 1999.

BIGRAS-POULIN, M.; THOMPSON, R. A.; CHRIEL, M.; MORTENSEN, S.; GREINER, M. Network analysis of Danish cattle industry trade patterns as an evaluation of risk potential for disease spread. Preventive Veterinary Medicine, v. 76, n. 1-2, p. 11-39, 2006.

BIGRAS-POULIN, M.; BARFOD, K.; MORTENSEN, S.; GREINER, M. Relationship of trade patterns of the Danish swine industry animal movements network to potential disease spread. Preventive Veterinary Medicine, v. 80, n. 2-3, p.143-165, 2007.

CSARDI, G.; NEPUSZ, T. The igraph software package for complex network research, InterJournal, Complex Systems, 1695, 2006. Disponível em: <http://igraph.sf.net/>. Acessado em: 08 jun. 2011.

GRISI-FILHO, J. H. H.; AMAKU, A.; OSSADA, R.; FERREIRA, F. Different methods to generate scale-free networks and their effects on the network shape. In: DYNAMICS DAYS SOUTH AMERICA, 2010, São José dos Campos. Book of abstracts and program, 2010. p. 165-166.

JEONG, H. Complex scale-free networks. Physica A, v. 321, n. 1-2, p. 226-237, 2003.

KALISKY, T.; COHEN, R.; HAVLIN, S. Tomography and Stability of Complex Networks. Lecture Notes in Physics, v. 650, p. 3-34, 2004.

LINDQUIST, J.; MA, J.; VAN DEN DRIESSCHE, P.; WILLEBOORDSE, F. H. Effective degree network disease models. Journal of Mathematical Biology, v. 62, n. 2, p. 143-164, 2011.

LLOYD, A. L.; MAY, R. M. How viruses spread among computers and people. Science, v. 292, n. 5520, p. 1316-1317, 2001.

MATO GROSSO. Secretaria de Assuntos Fundiários do Estado de Mato Grosso - Instituto de Defesa Agropecuária do Estado de Mato Grosso - INDEA. Coordenadoria de Controle das Doenças dos Animais - CCDA. Banco digitalizado de informação da vacinação. Cuiabá: INDEA, 2008. 
MAY, R. M.; LLOYD, A. L. Infection dynamics on scale-free networks. Physical Review E, v. 64, n. 16, p. 066112-1-066112-4, 2001.

MEYERS, L. A. Contact network epidemiology: bond percolation applied to infectious disease prediction and control. Bulletin of the American Mathematical Society, v. 44, n. 1, p. 63-86, 2007.

NATALE, F.; GIOVANNINI, A.; SAVINI, L.; PALMA, D.; POSSENTI, L.; FIORE, G.; CALISTRI, P. Network analysis of Italian cattle trade patterns and evaluation of risks for potential disease spread. Preventive Veterinary Medicine, v. 92, n. 4, p. 341-350, 2009.

NEGREIROS, R. L. Caracterização e análise da rede de movimento de bovinos no Estado de Mato Grosso. 2010. 121 p. Tese (Doutorado em Ciências) - Faculdade de Medicina Veterinária e Zootecnia, Universidade de São Paulo, São Paulo, 2010.

NEWMAN, M. E. J. Networks: an introduction. Oxford: Oxford University Press, 2010. 772 p.

OSSADA, R.; AMAKU, M.; GRISI-FILHO, J. H. H.; FERREIRA, F. Modelagem da dinâmica de doenças infecciosas em redes complexas. In: CNMAC (CONGRESSO NACIONAL DE MATEMÁTICA APLICADA E COMPUTACIONAL), 33., 2010, Águas de Lindóia. 2010. p. 1038-1039.

PASTOR-SATORRAS, R.; VESPIGNANI, A. Epidemic spreading in scale-free networks. Physical Review Letters, v. 86, n. 14, p. 3200-3203, 2001.

R DEVELOPMENT CORE TEAM. R: a language and environment for statistical computing. Vienna, Austria: 2010. R Foundation for Statistical Computing. Disponível em: $<$ http://www.R-project.org/>. Acessado em: 08 jun. 2011.

VERNON, M. C.; KEELING, M. J. Representing the UK's cattle herd as static and dynamic networks. Proceedings of the Royal Society B, v. 276, n. 1656, p. 469-476, 2009.

WASSERMAN, S.; FAUST, K. Social network analysis: methods and applications. Cambridge: Cambridge University Press, 1994. 825 p.

WOOLHOUSE, M. E. J.; SHAW, D. J.; MATTHEWS, L.; LIU, W-C.; MELLOR, D. J.; THOMAS, M. R. Epidemiological implications of the contact network structure for cattle farms and the 20-80 rule. Biology Letters, v. 1, n. 3, p. 350-352, 2005. 